\title{
RF and microwave photonic high bandwidth signal processing based on Kerr micro-comb sources
}

\author{
Mengxi Tan, ${ }^{1}$ Xingyuan Xu, ${ }^{1,}$ Jiayang Wu, ${ }^{1}$ Roberto Morandotti, ${ }^{2}$ Arnan Mitchell, ${ }^{3}$ \\ and David J. Moss ${ }^{1}$
}

\footnotetext{
${ }^{1}$ M. Tan, X. Xu, J. Wu, and D. J. Moss are with the Optical Sciences Centre, Swinburne University of Technology, Hawthorn, VIC 3122, Australia. (Corresponding e-mail: dmoss@ swin.edu.au).

${ }^{2} \mathrm{R}$. Morandotti is with INRS -Énergie, Matériaux et Télécommunications, 1650 Boulevard Lionel-Boulet, Varennes, Québec, J3X 1S2, Canada; visiting professor, Frontier Sciences, University of Electronic Science and Technology of China, Chengdu 610054, China.

${ }^{3}$ A. Mitchell is with the School of Engineering, RMIT University, Melbourne, VIC 3001, Australia.

* X. Xu current address: Electro-Photonics Laboratory, Dept. of Electrical and Computer Systems Engineering, Monash University, VIC3800, Australia.
}

\begin{abstract}
Integrated Kerr micro-combs are a powerful source for generating multiple wavelength channels to achieve photonic radio frequency (RF) and microwave signal processing, particularly in the context of transversal filters. They offer competitive advantages including a compact device footprint, high versatility, large numbers of wavelengths, and wide Nyquist bands. Here, we review recent progress on Kerr micro-combbased photonic RF and microwave high bandwidth temporal signal processing, including integral and fractional Hilbert transforms, differentiators as well as integrators. The strong potential of optical micro-combs for RF photonic applications in terms of functions and integrability is also discussed.
\end{abstract}

Index Terms-Microwave photonics, micro-ring resonators.

\section{INTRODUCTION}

Nonlinear optics for all-optical signal processing has proven to be extremely powerful, particularly when implemented in photonic integrated circuits based on highly nonlinear materials such as silicon [1-3]. All optical signal processing functions include all-optical logic [4], demultiplexing from 160Gb/s [5] to over 1Tb/s [6], optical performance monitoring via slow light [7,8], all-optical regeneration [9,10], and others [11-16]. Complementary metal oxide semiconductor (CMOS) compatible platforms are centrosymmetric where the $2^{\text {rd }}$ order nonlinear response is zero, and so nonlinear devices in these platforms have been based on $3^{\text {rd }}$ order nonlinear susceptibility including third harmonic generation [11,17-21] and the Kerr nonlinearity $\left(n_{2}\right)$ [1,2]. The efficiency of Kerr based all-optical devices depends on the waveguide nonlinear parameter $(\gamma)$. Although silicon can achieve extremely high values of $\gamma$, it suffers from high nonlinear losses due to two-photon absorption (TPA) and the resulting free carriers [2]. Even if the free carriers are eliminated by $\mathrm{p}-\mathrm{i}-\mathrm{n}$ junctions, silicon's relatively poor intrinsic nonlinear figure of merit $\left(\mathrm{FOM}=n_{2} /(\beta \lambda)\right.$, where $\beta$ is the TPA and is $\lambda$ the wavelength) of around 0.3 in the telecom band is far too low to achieve high performance. While TPA can be turned to advantage for some all-optical functions [22-24], for the most part silicon's low FOM in the telecom band is a limitation. This has motivated research on a range of alternate nonlinear platforms highlighted by the chalcogenide glasses [25-34]. However, while offering many advantages, these platforms are not compatible with the CMOS silicon computer chip industry.

In 2008 new CMOS compatible platforms for nonlinear optics, including silicon nitride [35, 36] and high-index doped silica glass (Hydex) [37-47] were introduced that displayed negligible nonlinear absorption in the telecom band together with a moderate nonlinear parameter, resulting in an extremely high nonlinear figure of merit. Following the first report of micro-resonator based Kerr frequency comb sources in 2007 [48], the first integrated CMOS compatible optical parametric oscillators were reported in 2010 [36, 37], and since then this field has exploded [47]. Integrated optical Kerr frequency comb sources, or "micro-combs" have come into focus as a fundamentally new and powerful tool due to their ability to provide highly coherent multiple wavelength channels with a very high degree of wavelength spacing control, from a single source. They are generated by optical parametric oscillation in monolithic micro-ring resonators (MRRs) and offer significant advantages over more traditional multi-wavelength sources. Many cutting- 
edge applications have been demonstrated based on CMOS-compatible micro-combs, ranging from filter-driven modelocked lasers [49-52] to quantum physics [53-61]. Most recently, Kerr micro-combs have demonstrated their enormous potential as sources for ultrahigh bandwidth coherent optical fiber communications [62-64], optical neural networks [65-67], optical frequency synthesis [68], and have been reviewed extensively [47, 69 - 76]. The success of these new CMOS platforms as well as other CMOS platforms such as amorphous silicon [77] and silicon rich silicon nitride (SRSN) [78] arises from a combination of their low linear loss, moderate to high nonlinearity and low or even negligible nonlinear loss (TPA).

All-optical signal processing has been particularly important for a wide range of RF photonic applications from radar systems to communications [79-128] because of the ultra-high RF bandwidths, low transmission loss, and strong immunity to electromagnetic interference (EMI), that it can deliver. There are a very wide variety of approaches to realizing photonic RF devices, including ones that map the optical filter response onto the RF domain, highlighted by on-chip (waveguide based) stimulated Brillouin scattering [88-95]. This approach has achieved extremely high performance in terms of RF resolution - as high as $32 \mathrm{MHz}$ - and a stopband rejection > $55 \mathrm{~dB}$. Another approach focuses on reconfigurable transfer functions for adaptive signal processing based on transversal filters [96-100] that operate by generating weighted and progressively delayed replicas of the RF signal in the optical domain and then combining them upon photo-detection. Transverse filters are capable of achieving arbitrary RF transfer functions by simply changing the tap weights, and so are attractive for the implementation of advanced adaptive and dynamic RF filters. Discrete laser arrays [101] and Bragg grating arrays or sampled gratings [103] have been employed to supply the needed taps. However, while offering many advantages, these approaches result in significantly increased complexity as well as reduced performance due to the limited number of available taps. Alternative approaches, including those based on optical frequency comb sources achieved by electro-optic (EO) or acousto-optic (AO) modulation [102,104,105], can help mitigate this problem, but they require cascaded high frequency modulators that in turn require high-frequency RF sources. Of these approaches, EO comb-based method has been in use the longest and have demonstrated many powerful functions.

Integrated Kerr micro-combs offer many advantages over more traditional multi-wavelength sources for RF applications. They can greatly increase the capacity of communications systems and allow the processing of RF spectra for a very wide range of advanced signal processing functions [107-128]. They have the advantage of being able to generate frequency combs with much higher spacings than electro-optic combs. In fact, EO combs and micro-combs are in many ways complementary - the former focusing on finer wavelength spacings from 10's of MHz to $10-20$ $\mathrm{GHz}$, while the integrated micro-combs typically have much wider spacings from 10-20 GHz up to 100's of GHz and even into the terahertz regime. Wider comb spacings yield much wider Nyquist zones for larger RF bandwidths, whereas finer spacings potentially can yield much larger numbers of wavelengths or RF "taps", at the expense of much more limited Nyquist zones and hence RF bandwidths. Micro-combs have the potential to provide a much higher number of wavelengths, an ultra-large large free spectral range (FSR), as well as greatly reduced footprint and complexity. In particular, for RF transversal filters the number of wavelengths dictates the available channel number of RF time delays, and thus with micro-combs, the performance of RF filters [85, 121] and other systems such as beamforming devices [112] can be greatly enhanced in terms of the quality factor and angular resolution, respectively. In addition, other approaches to filtering such as RF bandwidth scaling [125] have a given bandwidth for each wavelength channel, and so the total operation bandwidth (the maximum RF signal bandwidth that can be processed) will depend on the number of wavelengths, and so will be greatly enhanced with micro-combs.

Recently [121], we reviewed RF and microwave spectral filters based on transversal filtering and bandwidth scaling methods based on Kerr micro-combs. Here, we focus on RF and microwave high bandwidth temporal signal processing based on integrated Kerr micro-comb devices, for integral and fractional order Hilbert transformers, differentiators, as well as for integrators. We discuss the trade-offs between using very widely spaced micro-combs at 200GHz [109-111] with recently reported record low FSR spaced micro-combs at $49 \mathrm{GHz}$, based on soliton crystals [122-128]. We highlight their potential and future possibilities, contrasting the different methods and use of the differently spaced micro-combs. While RF transversal filters based on $200 \mathrm{GHz}$ Kerr micro-combs have achieved a high degree of versatility and dynamic reconfigurability, their relatively large comb spacing arising from the large FSR of $\sim 1.6 \mathrm{~nm}$ $(\sim 200 \mathrm{GHz})$ restricts the number of taps to $<21$ within the $30 \mathrm{~nm}$ wide bandwidth of the C-band. This is an important 
consideration since this approach requires optical amplifiers and optical spectral shapers which are more readily available in the C-band. The limitation in the number of taps has restricted the performance of micro-comb based transversal RF filters in terms of frequency selectivity, bandwidth, and dynamic versatility. In order to improve on this, we demonstrated micro-comb-based photonic RF transversal filters with record high numbers of taps - up to 80 wavelengths over the C-band [117]. This is the highest number so far reported for micro-comb-based RF transversal filters, enabled by a record low $49 \mathrm{GHz}-\mathrm{FSR}$ integrated Kerr micro-comb source. While for filter applications [121] this resulted in $Q_{\mathrm{RF}}$ factors for RF bandpass filters of four times higher than for $200 \mathrm{GHz}$ combs, for temporal signal processing this also yields dramatic improvements in bandwidth and reconfigurability. Our results verify the feasibility of realizing high performance advanced adaptive RF transversal filters for high bandwidth signal processing with potentially reduced cost, footprint, and complexity than other solutions.

\section{INTEGRATED KERR MICRO-COMBS}

The generation of micro-combs is a complex process that relies on a combination of high nonlinear optical parameters, low linear and nonlinear loss as well as engineered dispersion. Diverse platforms have been developed for micro-combs [47] such as silica, magnesium fluoride, silicon nitride, and doped silica glass [47, 70, 85]. In 2008 [39] we reported efficient four-wave mixing at milliwatt power levels based on the $575 \mathrm{GHz}$ FSR devices that had a relatively low Q-factor of 60,000. This represented the first report of low power CW nonlinear optics in silica glass based MRRs. This was followed in 2010 by the first integrated micro-combs [37, 38] in Hydex and silicon nitride - the first optical micro-combs based on a fully integrated photonic chip platform, inspired by micro-combs in toroidal resonators [48]. Another breakthrough came with the report of [116, 117] integrated micro-combs with record low spacings of $<50 \mathrm{GHz}$ which greatly expanded the number of wavelengths over the telecommunications band to 80 or more. Apart from a record low FSR, these micro-combs operated via a different process to single soliton states [66$73]$, called soliton crystals $[129,130]$. Many other recent breakthroughs have been reported in micro-combs, such as ultralow pump power combs [131], dark solitons [132], laser-cavity solitons [133] and others [134-139].

The MRRs used in the work reviewed here were fabricated on a Hydex platform $[37,38]$ using CMOS compatible fabrication processes, with Q factors ranging from 60,000 to over 1.5 million and radii ranging from $\sim 592 \mu \mathrm{m}$ to $\sim 135$ $\mu \mathrm{m}$ and even smaller at $\sim 48 \mu \mathrm{m}$, corresponding to FSRs of $\sim 0.4 \mathrm{~nm}(\sim 49 \mathrm{GHz}), \sim 1.6 \mathrm{~nm}(\sim 200 \mathrm{GHz})$, and $\sim 4.5 \mathrm{~nm}$ $(\sim 575 \mathrm{GHz})$, respectively. The work on micro-combs was based on $200 \mathrm{GHz}$ (Fig. 1a) and 49GHz (Fig. 1b) FSR MRRs. First, high-index $(n=\sim 1.7$ at $1550 \mathrm{~nm}$ ) doped silica glass films were deposited using plasma enhanced chemical vapour deposition, then patterned by deep ultraviolet photolithography and etched via reactive ion etching to form waveguides with exceptionally low surface roughness. Finally, silica $(n=\sim 1.44$ at $1550 \mathrm{~nm})$ was deposited as an upper cladding. The device architecture typically uses a vertical coupling scheme where the gap (approximately 200nm) can be controlled via film growth - a more accurate approach than lithographic techniques. The advantages of our platform for optical micro-comb generation include ultra-low linear loss $\left(\sim 0.06 \mathrm{~dB} \cdot \mathrm{cm}^{-1}\right)$, a moderate nonlinear parameter $(\sim 233$ $\left.\mathrm{W}^{-1} \cdot \mathrm{km}^{-1}\right)$, and in particular negligible nonlinear loss up to extremely high intensities $\left(\sim 25 \mathrm{GW} \cdot \mathrm{cm}^{-2}\right)$. Due to the ultralow loss the MRR featured narrow resonance linewidths, corresponding to quality factors of up to 1.5 million (Fig. 1) for both MRRs. After packaging the devices with fiber pigtails, the through-port insertion loss was typically $1 \mathrm{~dB} /$ facet, to as low as $0.5 \mathrm{~dB} /$ facet, assisted by on-chip mode converters.

To generate micro-combs with the $200 \mathrm{GHz}$ devices, the continuous-wave (CW) pump power was typically amplified to over $\sim 30 \mathrm{dBm}$ and then the wavelength swept from blue to red near one of the TE resonances of the MRR near $\sim 1550 \mathrm{~nm}$. When the detuning between the pump wavelength and MRR's cold resonant wavelength became small enough such that the intracavity power reached a threshold, a modulation instability driven oscillation was initiated [47]. Primary combs were thus generated with the spacing determined by the MI gain peak - mainly a function of the intra-cavity power and dispersion. As the detuning was changed further, single-FSR spaced micro-combs eventually were generated. Achieving rigorous single soliton states in micro-combs via dissipative Kerr solitons (DKS), while well understood [66], is nonetheless challenging, although recent progress has been made [139]. It requires both frequency and amplitude sweeping in both directions in order to "capture" the soliton states out of the initially chaotic states. Our initial work on micro-comb applications was based on the $200 \mathrm{GHz}$ micro-combs operating in a partially coherent state that, while not exhibiting rigorous soliton behaviour, was nonetheless a relatively low noise state that avoided the chaotic regime [47] and was sufficient for microwave applications. The spectra shown in Figs. 1b and 1c 
are typical of the $200 \mathrm{GHz}$ combs in our initial microwave work, and clearly show that it is not a rigorous soliton state. Nonetheless they were successful at demonstrating a wide range of RF signal processing functions [85, 107, 109 -111].

Subsequently, we reported Kerr solitons with a record low FSR of 49GHz, based on a new class of soliton [129, 130] called soliton crystals. Soliton crystals result from mode crossings, in our case in the C-band, and are easier and more robust to generate than DKS states, and indeed even easier than the partially coherent $200 \mathrm{GHz}$ FSR comb states. They can be generated even with manual tuning of the pump laser wavelength, mainly because the internal cavity optical energy of the soliton crystal state is very close to that of the chaotic state. Therefore, when transitioning from chaos to the soliton crystal state, there is only a very small shift in internal energy and hence very little resonant wavelength jump. It is this "jump" in both internal energy and resonant wavelengths of the micro-resonator that makes DKS states more challenging. The efficiency of the soliton crystal states (energy in the comb lines relative to the pump wavelength) is also much higher than DKS states (the single soliton states in particular), for the same reason. There is a trade-off, though, in that soliton crystal spectra are not flat but exhibit characteristic "curtain-like" patterns. While this can require spectral flattening, it has not posed a fundamental barrier to achieving a wide range of high performance RF and microwave processing functions. Soliton crystals also result from mode-crossings in the resonator, which can be challenging to design, rather than simply requiring the anomalous dispersion that DKS states need, but this has not posed a barrier to fabricating many devices with high yield [64].

When generating soliton crystals, the pump laser was manually tuned across a resonance (TE polarized) and when the pump (with sufficient power) was aligned well enough with the resonance, a primary comb was generated, similar to the $200 \mathrm{GHz}$ states. In this case, however, as the detuning was changed further, distinctive 'fingerprint' optical spectra were observed (Fig. 2), arising from spectral interference between tightly packed solitons in the cavity - soliton crystals $[129,130]$. An abrupt step in the measured intracavity power (Fig. 2(b)) was observed at the point where these spectra appeared, as well as a dramatic reduction in the RF intensity noise (Fig. 2(c)). Together with the spectra shapes, these observations indicate soliton crystal formation [38], although to conclusively demonstrate this would need to perform time resolved pulse autocorrelation measurements. The key issue for the RF experiments was not the specific microcomb state with the low RF noise and high coherence, which were relatively straightforward to achieve through adiabatic pump wavelength sweeping. We found that it is not necessary to achieve a specific state, only that the chaotic regime [47] should be avoided. Nonetheless soliton crystals provide the lowest noise states of all micro-combs, and have formed the basis for a microwave oscillator with low phase-noise [120]. This is important since there are a much wider range of coherent low RF noise states that are more readily accessible than any specific soliton related state [47].

\section{RF TRANSVERSAL FILTER: THEORY AND EXPERIMENT}

The transfer function of a general transversal structure can be described as [85]

$$
F(\omega)=\sum_{k=0}^{M-1} h_{k} e^{-j \omega K T}
$$

Where $j=\sqrt{-1}, \omega$ is the angular frequency of the input RF signal, $M$ is the number of taps, $T$ is the time delay between adjacent taps, and $h_{k}$ is the tap coefficient of the $k_{t h}$ tap, which is the discrete impulse response of $F(\omega)$. The discrete impulse response can be calculated by performing the inverse Fourier transform of $F(\omega)$, and then temporally windowing it with a short cosine bell. The transversal structure, similar to the concept of finite impulse response digital filters, is a fundamental tool for photonic RF signal processing. With the proper design of the tap weights $h_{k}$ on each wavelength channel, any transfer function can be arbitrarily realized for different signal processing functions such as Hilbert transformers, differentiators and integrators. The Nyquist frequency of the response function is given by $f_{N y q u i s t}$ $=1 / 2 T$. Figure 3 shows a schematic diagram of a transversal filter based on a multi-wavelength source, indicating that the core of the structure consists of two operations: broadcast and then delay. The input RF signal is first broadcast onto equally spaced wavelength channels via optoelectronic modulation to generate multiple replicas of the RF input. The replicas are then transmitted through a dispersive medium to acquire wavelength-sensitive delays. Second-order dispersion, which yields a linear relationship between the wavelength and the delay, is generally employed to progressively separate the replicas. The delayed signals can be either individually converted into electronic signals or summed upon photodetection. The former can be implemented through wavelength demultiplexers and photodetector arrays to realize RF true time delays [116], while the latter can serve as transversal filters for RF signal processing [85]. 
The large number of wavelengths offered by the micro-combs provides significant advantages for enhancing the performance of transversal signal processors. The number and amplitude of progressively delayed RF replicas (taps) determines the performance of the system. By setting the tap coefficients, a reconfigurable transversal filter with diverse transfer functions can be achieved, including Hilbert transformers [107, 126], differentiators [109,127], bandpass filters [111,117, 121], integrators [123], waveform generators [128] and more.

Figure 4 shows the experimental setup for transversal filter systems based on integrated Kerr optical comb sources with FSRs of $200 \mathrm{GHz}$ (Fig. 4(a)) and 49GHz (Fig. 4(b)). In both cases the micro-combs, generated by the on-chip MRR, are amplified and fed to either one $(200 \mathrm{GHz}$ comb) or two $(49 \mathrm{GHz}$ comb) waveshapers for channel equalization and weighting. The second waveshaper for the $49 \mathrm{GHz}$ FSR comb is sometimes used to pre-flatten the non-uniform comb spectra of the soliton crystals. The power of each comb line is adjusted by the waveshaper to achieve the required tap coefficients. To increase the accuracy, real-time feedback control paths are used to read and shape the power of the comb lines. The comb lines are then divided into two paths according to the sign of the tap coefficients, and then into a $2 \times 2$ balanced Mach-Zehnder modulator (MZM) biased at quadrature for the $200 \mathrm{GHz}$ micro-comb experiments. The $2 \times 2$ balanced MZM could simultaneously modulate the input RF signal on both positive and negative slopes, thus yielding replicas of the input RF signal with phase and tap coefficients having either algebraic sign. For experiments with the 49GHz FSR microcomb, both positive and negative taps were achieved by separating the wavelength channels according to the designed tap coefficients and then fed into a balanced photodetector (Finisar BPDV2150R) (Figure $4 b)$.

For the $200 \mathrm{GHz}$ comb based system, the signal modulated by the MZM then went through $2.122-\mathrm{km}$ of standard single mode fibre (SMF), where the dispersion was $\sim 17.4 \mathrm{ps} /(\mathrm{nm} \cdot \mathrm{km})$, corresponding to a time delay $T$ of $\sim 59 \mathrm{ps}$ between adjacent taps (with the channel spacing of the time delay lines equalling to the FSR of the micro-combs), yielding a Nyquist frequency of $\sim 8.45 \mathrm{GHz}$. The bandwidths of the systems were determined by the Nyquist frequency, which could be easily enlarged by decreasing the time delay and, owing to the large FSR of the compact MRR, could potentially reach over $\sim 100 \mathrm{GHz}$. Finally, the weighted and delayed taps were combined upon detection and converted back into RF signals via a high-speed photodetector (Finisar, $40 \mathrm{GHz}$ bandwidth) at the output.

For the 49GHz-FSR MRR, the signal went through $\sim 5-\mathrm{km}$ of standard SMF to provide the progressive tap delays. The dispersion of the fibre was the same $(17 \mathrm{ps} / \mathrm{nm} / \mathrm{km})$, yielding a time delay $T$ of $\sim 34.8 \mathrm{ps}$ between adjacent taps. This resulted in an operation bandwidth (Nyquist frequency, half of $F S R_{\mathrm{RF}}$ ) of $\sim 14.36 \mathrm{GHz}$ for the transversal filter. Again, this can be enlarged by decreasing the time delay (e.g., using a shorter spool of SMF), at the expense of a reduced tuning resolution. However, the maximum operational bandwidth of the transversal filter is limited by the comb spacing. For the $49 \mathrm{GHz}$ comb, significant crosstalk between adjacent wavelength channels occurs beyond 24.5 $\mathrm{GHz}$ - half of the micro-comb's spacing. This issue can be addressed by employing a micro-comb source with a larger comb spacing, although at the expense of providing fewer comb lines/taps across the C-band). Note that although standard optical fibre is often used to produce the dispersive delays, this can readily be done via other means. Typically, only about 2-km of SMF, yielding a dispersion of $34 \mathrm{ps} / \mathrm{nm}$, is needed. This is within the range of multi-channel tunable dispersion compensators [140-143], which can also be designed with a dispersion slope offset. This approach would not only yield a compact and latency free delay but would enable tunability to adjust the Nyquist zone. In the following sections, we will review progress made in high bandwidth temporal RF and microwave signal processing functions including Hilbert transform, differentiation and integration based on Kerr micro-combs.

\section{HILBERT TRANSFORMS}

The Hilbert transform (HT) is a fundamental signal processing operation with wide applications in radar systems, signal sideband modulators, measurement systems, speech processing, signal sampling and many others [144]. Standard integral HTs perform a $\pm 90^{\circ}$ phase shift around a central frequency, with an all-pass amplitude transmission. Fractional Hilbert transform (FHT) provides a variable phase shift for applications such as hardware keys [145, 146], secure single sideband communications [145], and in forming an image that is edge enhanced relative to the input object, where they can select which edges are enhanced and the degree of edge enhancement [147]. Electronic fractional Hilbert transformers are limited in operation bandwidth [145, 148], whereas photonic technologies offer broadbandoperation as well as strong immunity to EMI. Hilbert transformers based on free-space optics have been demonstrated [146, 149] that achieve high performance but are bulky and complex. Phase-shifted fibre Bragg gratings based Hilbert transformers have bandwidths of a few $100 \mathrm{GHz}$ [150-153] but only yield precise FHT for signals with specific bandwidths, fixed fractional orders, and only operate on the complex optical field (not RF signal). This also holds for integrated reconfigurable microwave processors [154] as well Bragg grating approaches [155]. In practice, the FHT of 
the RF and microwave signals - not the complex optical fields - is needed for RF measurement and signal reshaping [156-163].

Integrated Hilbert transformers based on microring or disc resonators, Bragg gratings in silicon, and integrated InPInGaAsP photonic chips yield compact devices with high stability and mass-producibility [154, 155, 159-161]. Nevertheless, they only operate on the complex optical field and generally only provide a limited phase shift tuning range. Transversal filter approaches offer high reconfigurability [162, 163] but need many discrete lasers, thus increasing system size, cost, and complexity, and limiting the number of taps and therefore performance. Optical frequency combs (OFCs) provide a single high-quality source for many wavelengths, including mode-locked lasers [164], electro-optical modulators [165], and micro-resonators [166]. Of these, Kerr micro-combs generated by microresonators provide many wavelengths with greatly reduced footprint.

The spectral transfer function of a general fractional Hilbert transformer can be expressed as [80, 126]:

$$
H_{P}(\omega)=\left\{\begin{array}{c}
e^{-j \varphi}, \text { if } 0 \leq \omega<\pi \\
e^{j \varphi}, \text { if }-\pi \leq \omega<0
\end{array}\right.
$$

where $j=\sqrt{-1}, \varphi=P \times \pi / 2$ is the phase shift, with $P$ denoting the fractional order. As can be seen from Eq. (2), an FHT can be regarded as a phase shifter with $\pm \varphi$ phase shifts around a centre frequency $\omega$, becoming a classical integral HT when $P=1$. The corresponding impulse response is a continuous hyperbolic function:

$$
h_{P}(t)=\left\{\begin{array}{c}
\frac{1}{\pi t}, t \neq 0 \\
\cot (\varphi), t=0
\end{array}\right.
$$

The hyperbolic function is truncated and sampled in time by discrete taps. The sample spacing $\Delta t$ determines the null frequency $f_{\mathrm{c}}=1 / \Delta t$. The order of the FHT is continuously tunable by only adjusting the coefficient of the tap at $t=0$ while keeping the same coefficients for the other taps [146].

The normalized power of each comb line, needed to achieve a Hilbert Transform, is given by:

$$
p_{n}=\frac{1}{\pi|n-N / 2+0.5|}
$$

where $N$ is the number of comb lines, or filter taps, used in the filters, and $n=0,1,2, \ldots, N-1$ is the comb index.

In 2015, the first Kerr micro-comb based Hilbert transformers was demonstrated, with up to 20 taps [107] from a $200 \mathrm{GHz}$ comb that achieved a record RF bandwidth (5 octaves). Figures 5(a) and 5(b) show the shaped optical microcombs measured using an optical spectrum analyzer for the 12 tap and 20 tap filter cases, respectively. The target powers at all wavelengths are also shown in Figs. 5(a) and 5(b) as green crosses. The waveshaper was successfully used to shape the powers of all comb lines to within $+/-0.5 \mathrm{~dB}$ of the target powers. This could be easily achieved since the waveshaper resolution $(10 \mathrm{GHz})$ was much smaller than the comb spacing $(200 \mathrm{GHz})$. Unused comb lines were attenuated below the noise floor. Once the comb lines were attenuated in order to provide the correct tap coefficients of the impulse response associated with a HT, the system RF frequency response was then characterized. A vector network analyzer (VNA) was used to measure the system RF amplitude and phase frequency response. Figure 6a shows the measured RF amplitude frequency response of the photonic Hilbert transform filters for 12, 16 and 20 taps, respectively, which all exhibit expected behavior. All three filters have the same null frequency at $16.9 \mathrm{GHz}$, corresponding to the tap spacing of $\Delta t=1 / f_{c}=59 \mathrm{ps}$. This spacing matches the difference in delay between the comb lines, equal to the ring $F S R=1.6 \mathrm{~nm}$, produced by propagation through a $2.122-\mathrm{km}$ long spool of SMF with a dispersion parameter $D=17.4 \mathrm{ps} / \mathrm{nm} / \mathrm{km}$ ). The null frequency could be controlled by using a different fiber length to adjust the tap spacing. All filters show $<3 \mathrm{~dB}$ amplitude ripple. As expected, increasing the number of filter taps increases the filter bandwidth. With a 20-tap filter, the Hilbert transformer exhibited a 3-dB bandwidth extending from $16.4 \mathrm{GHz}$ down to $0.3 \mathrm{GHz}$, corresponding to more than 5 octaves. It is possible to increase this bandwidth by using more comb lines in the filter. In those experiments, only a small portion of the generated comb lines was actually used to realize the filter taps. The number of filter taps that could be achieved was actually limited by the bandwidth of the waveshaper and the gain bandwidth of the erbium-doped fiber amplifier (EDFA). If desired, it would also be possible to reduce the amplitude ripple within the passband by apodizing the tap coefficients from the ideal hyperbolic function. 
Figure 6(b) shows the measured phase response of filters with different numbers of taps, showing very similar responses. Each shows a relatively constant phase of near $-90^{\circ}$ within the passband. There are some deviations from the ideal $-90^{\circ}$ phase at frequencies close to zero and particularly for the null frequency $f_{c}=16.9 \mathrm{GHz}$.

Recently, [126] Tan et. al demonstrated a fractional Hilbert transformer based on a Kerr micro-comb with a significantly finer comb spacing of $49 \mathrm{GHz}$ that provided a much larger number of comb lines ( 80 in the $\mathrm{C}$ band). By programming and shaping the comb lines according to calculated tap weights, FHT with very broad bandwidths and arbitrary fractional orders with phase shifts of $15^{\circ}, 30^{\circ}, 45^{\circ}, 60^{\circ}, 75^{\circ}$, and $90^{\circ}$ was achieved, corresponding to fractional orders of $0.17,0.33,0.5,0.67,0.83$, and 1 , respectively. Operation over $>5$ octaves from $480 \mathrm{MHz}$ to $16.45 \mathrm{GHz}$ for the $90^{\circ}$ phase-shift FHT and \pm 0.07 rad phase variation within the 3 - $\mathrm{dB}$ bandwidth was achieved. System demonstrations of a real-time FHT for Gaussian pulse input signals were also performed. The good agreement with theory confirmed this approach as an effective way to implement high-speed reconfigurable FHTs with reduced footprint, lower complexity, and potentially lower cost.

In those experiments a maximum of 17 wavelengths were used. The space between the 8th and 9th taps and 9th and 10th taps was $0.8 \mathrm{~nm}$, while the remaining spacings were $1.6 \mathrm{~nm}$, done in order to maximize the Nyquist zone. The delay line, $2.1-\mathrm{km}$ of single mode fibre $(\beta=\sim 17.4 \mathrm{ps} / \mathrm{nm} / \mathrm{km})$ provided a time delay between channels of $\tau=\mathrm{L} \times \beta$ $\times \Delta \lambda=\sim 29.4 \mathrm{ps}$, corresponding to a FSR $\mathrm{RF}_{\mathrm{F}}$ of $1 / 2 \tau=\sim 17 \mathrm{GHz}$. An increased bandwidth can be obtained with a shorter fibre. The RF amplitude and phase frequency response was characterized with a VNA.

The theoretical amplitude and phase response of FHT with $15^{\circ}, 30^{\circ}, 45^{\circ}, 60^{\circ}, 75^{\circ}$, and $90^{\circ}$ phase shifts are shown in Figs. 7 (a)-(f) solid black curves for varying numbers of taps. The corresponding fractional orders are 0.17, 0.33, 0.5, 0.67, 0.83, and 1, respectively. Figures 8(a) and (b) show the RF amplitude and phase frequency response for a $45^{\circ}$ phase shift with 5, 9, 13 and 17 taps, respectively. As expected, the bandwidth increases with the number of taps. With 17 taps, the operating 3-dB bandwidth for base-band RF signals was $0.48 \mathrm{GHz}$ to $16.45 \mathrm{GHz}$ - more than 5 octaves. For a standard $90^{\circ} \mathrm{HT}$ the soliton crystal micro-comb provided more than 40 taps, thereby reducing the rootmean-squared error (RMSE) and amplitude ripple within the passband [126]. As seen in Fig. 8 (c), the theoretical 3$\mathrm{dB}$ bandwidth increases rapidly with the number of taps up to 17 taps, after which it levels off, so that 17 is close to the optimum number of taps.

Figures 9 (ii)-(iii) present the simulated (red dashed curves) and measured (blue solid lines) transmission response magnitude and phase. The normalized frequency and phase response of the fractional Hilbert transformer with variable orders from $0.166-0.833$ (phase shifts $15^{\circ}-90^{\circ}$ ) are shown. The amplitude variations are < $3 \mathrm{~dB}$ between $480 \mathrm{MHz}$ and $16.45 \mathrm{GHz}$ and the phase variations are about $\pm 0.07 \mathrm{rad}$ within the 3 - $\mathrm{dB}$ passband. Fig. 9 (iv) shows a pulsed waveform after processing by the fractional Hilbert transformer along with the simulated results (Fig. 9 (iv), red dashed curves). The detailed performance parameters are listed in Table I of Ref. [126]. As can be seen, the measured curves closely match the theoretical counterparts, achieving good performance that agrees well with theory.

By comparison, the 49-GHz FSR combs yielded superior performance over the 200-GHz comb-based transformer in terms of bandwidth, because of the larger number of available taps. Nonetheless, we note that it is not always a case of a smaller FSR comb being better. The $200-\mathrm{GHz}$ devices are capable of reaching RF bandwidths, particularly in tunability, approaching $100 \mathrm{GHz}$, or the Nyquist zone, whereas the $49 \mathrm{GHz}$ devices are limited to about $25 \mathrm{GHz}$.

\section{DIFFERENTIATION}

As a key basic function in RF signal processing systems, differentiation has wide applications in ultra-wideband (UWB) generation, RF spectrum analysis, and RF filters [167-171]. Significant progress has been made on RF integral differentiators via photonics technologies [172-184] using phase [172,173] and cross-phase modulation (XPM) [177179] methods, frequency discriminators and cross-gain modulation in semiconductor optical amplifiers (SOA) [176]. Fractional differentiation has many more unique and powerful applications $[127,182,183]$ and has played an important role in mechanics, electricity, chemistry, biology, and economics. Perhaps its most important applications have been in image edge detection, control theory, and mechatronics [182,183]. Despite this, however, photonic based fractional differentiators have received comparatively little attention.

Many photonic integral and fractional differentiators have focused on the derivative of the complex optical field, rather than the RF signal. A photonic differentiator based on a dual-drive MZM together with an RF delay line, [174] 
although successful, was limited in processing speed by the bandwidth of the RF delay line. Photonic differentiators based on optical filters [175] feature high speeds of up to 40-Gb/s, although they only work for a fixed (and typically integral) differentiation order and lack reconfigurability. To implement highly reconfigurable differentiators, transversal schemes have been investigated using discrete laser arrays [169, 180, 181], although at the expense of significantly increased system size, cost, and complexity, limiting the number of available taps and thus the processing performance. Using a single source that can simultaneously generate a large number of high-quality wavelength channels would be highly advantageous.

Recently [109], a photonic RF differentiator with 8 taps using a 200-GHz FSR micro-comb source was demonstrated and achieved the $1^{\text {st }}, 2^{\text {nd }}$, and $3^{\text {rd }}$ order differentiation operating at RF bandwidths up to $17 \mathrm{GHz}$ with a potential bandwidth of $100 \mathrm{GHz}$.

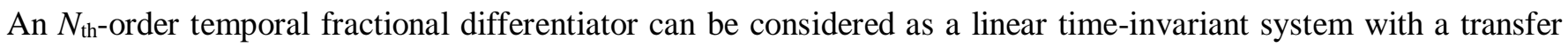
function given by [168]

$$
H_{N}(\omega) \propto(j \omega)^{N}
$$

where $j=\sqrt{-1}, \omega$ is the RF angular frequency, and $N$ is the differentiation order which can be fractional and even complex $[184,185]$. According to the above transfer function, the amplitude response of a temporal differentiator is proportional to $|\omega|^{N}$, while the phase response has a linear profile, with a phase jump of $N \pi$ at null frequencies.

Using the transversal filter approach towards the implementation of RF photonic differentiators yields a finite set of delayed and weighted replicas of the input RF signal in the optical domain which is combined upon detection. The transfer function from Eq. (5) becomes

$$
H_{M}(\omega)=\sum_{n=0}^{M-1} a_{n} e^{-j \omega n T}
$$

where $M$ is the number of taps, $a_{n}$ is the tap coefficient of the $n$-th tap, and $T$ is the time delay between adjacent taps. It should be noted that the differentiator design based on Eq. (6) is an intensity differentiator, i.e., the output RF signal after being combined upon detection yields an exact differentiation of the input RF signal, in contrast to field differentiators that yield the derivative of complex optical fields [167,171,153,154,185-187].

The first report of a micro-comb based differentiator [109] demonstrated integral $1^{\text {st }}, 2^{\text {nd }}$, and $3^{\text {rd }}$ order intensity differentiators with a 200GHz FSR Kerr micro-comb with 8, 6, and 6 taps, respectively, with a dynamic range, determined by the EDFA, of $\sim 30 \mathrm{~dB}$. More taps are needed when the differentiation order increases, and for a fixed number of taps, increasing the order of differentiation also increases the required power dynamic range. In order to get better performance with a limited number of taps, the operation bandwidth of the integral differentiators was decreased to half of the Nyquist frequency. To implement the temporal differentiator in Eq. (5), tap coefficients in Eq. (6) were calculated based on the Remez algorithm [188]. The calculated tap coefficients for $1^{\text {st }}, 2^{\text {nd }}$, and $3^{\text {rd }}$ order differentiation are listed in Table I of [109]. Since the $200 \mathrm{GHz}$ Kerr comb was not flat, real-time feedback control was used to increase the accuracy of the comb shaping. The corresponding amplitude and phase responses of the $1^{\text {st }}, 2^{\text {nd }}$, and $3^{\text {rd }}$ orders differentiators as a function of the number of taps are plotted in Figs. 10 (a)-(c). When the number of taps is increased, it is clear that the discrepancies between the amplitude responses of the transversal filters and the ideal differentiators are improved for all the three orders, whereas the phase response of the transversal filters is identical to that of the ideal differentiators regardless of the number of taps.

The shaped optical combs in Figs. 11 (b)-(d) show a good match between the measured comb lines' power (red solid line) and the calculated ideal tap weights (green cross). Figures. 12 (a-i), (b-i), and (c-i) show the measured and simulated amplitude responses of the differentiators. The corresponding phase response is depicted in Figs. 12 (a-ii), (b-ii), and (c-ii) where it can be seen that all three differentiators agree well with theory. The FSR of the RF response spectra was $\sim 16.9 \mathrm{GHz}$, consistent with the time delay between adjacent taps. By adjusting the FSR of the transversal filter by varying the dispersive fibre or programming the tap coefficients, a variable operation bandwidth for the intensity differentiator can be achieved, which is advantageous for diverse requirements of different applications.

System demonstrations of real-time signal differentiation are also performed for Gaussian input pulses with a fullwidth at half maximum (FWHM) of $\sim 0.12 \mathrm{ns,}$ as shown in Fig. 13 (a). The waveforms of the output signals after differentiation are shown in Figs. 13 (b)-(d) (blue solid curves), together with the ideal differentiation results (red 
dashed curves). The practical Gaussian pulse in Fig. 13 (a) is used as the input RF signal for the simulation. The measured curves closely match their theoretical counterparts, indicating good agreement between experimental results and theory. Unlike the field differentiators [167,171,153,154,185-187], temporal derivatives of intensity profiles can be observed, indicating that intensity differentiation was successfully achieved. For the first-, second-, and third-order differentiators, the calculated RMSE between the measured and the theoretical curves are $\sim 4.15 \%, \sim 6.38 \%$, and $\sim 7.24 \%$, respectively.

Subsequently, Tan et.al reported [127] the first fractional-order photonic based RF differentiator that operated directly on the RF signal, rather than the complex optical field. It was based on a 49-GHz-FSR Kerr micro-comb source, which provided a large number of comb lines ( 80 in the $\mathrm{C}$ band), resulting in a broad operation bandwidth of 15.49 GHz. By programming and shaping the comb lines according to calculated tap weights, reconfigurable arbitrary fractional orders ranging from 0.15 to 0.9 were demonstrated. System demonstrations of real-time fractional differentiation of Gaussian input pulses were also performed. The good agreement between theory and experiment confirmed this approach as an effective way to implement high-speed reconfigurable fractional differentiators with reduced footprint, lower complexity, and potentially lower cost.

The success of the differentiator resulted from both the use of soliton crystals combined with the low FSR of 48.9 $\mathrm{GHz}$, resulting in 80 wavelengths, or taps in the C-band. The successful results stemmed from the soliton crystal's extremely robust and stable operation and generation and as well as its much higher intrinsic efficiency, all of which are enabled by an integrated CMOS-compatible platform. A key advantage of that work was the large number of taps provided by the micro-combs. The relationship between the number of taps and the performance of the fractional differentiator was investigated. Figure 14 shows the transfer function of the fractional differentiator for six different orders. As can be seen, the operational bandwidth of the differentiator-within which the slope coefficients of the simulated and ideal amplitude responses match closely-increases with the number of taps. Figure 15 (a) shows that the number of taps is critical to the operation bandwidth of fractional differentiators, which is manifested experimentally for the fractional order $=0.45$ (Figs. 15 (b) and (c)). When the number of taps is increased to 27, the bandwidth of the fractional differentiator reached $15.49 \mathrm{GHz}$, occupying $>91 \%$ of the Nyquist band. In the experiment, up to 29 combs lines were employed to generate the taps. To increase the number of taps, the optical signal-to-noise ratio, which was subject to the optical amplifiers' noise, should be optimized. The shaped comb spectra are shown in Fig. 16. The optical power for each comb line matched the designed tap coefficients, verifying the success of our comb shaping procedure. The transmission response of the fractional differentiator was characterized using a VNA. The power response of the differentiator showed a bandwidth ranging from DC to $15.49 \mathrm{GHz}$. As shown in Fig. 17, the range of fractional orders was due to the close agreement between theory and experimental in terms of the slope coefficients of the power response and phase shift in the phase response. The performance of the fractional differentiator was further verified using a baseband RF Gaussian pulse generated by an arbitrary waveform generator featuring an FWHM of 200 ps (Fig. 18). The good agreement with theory reflects the range of orders that are achievable with our approach to realizing a reconfigurable fractional differentiator.

\section{INTEGRATION}

Temporal integration is another fundamental function in signal processing systems. In contrast to electrical integrators that are subject to the electronic bandwidth bottleneck, photonic techniques offer a broad bandwidth, strong immunity to EMI, as well as low loss [79,80,189]. Significant work has been reported on photonic integrators, such as those based on gratings [190-192] and micro-ring resonators (MRRs) [15,42,193]. These approaches achieve optical signal integration with a time feature as fast as 8 ps [193] and a large time-bandwidth product with high-Q resonant structures. However, these approaches still face limitations for RF signal processing. They are not adjustable in temporal resolution or length of integration window, thus lacking the flexibility of processing RF signals with different bandwidths and integration time windows. In addition, those approaches are designed to process optical signals - they cannot directly process RF signals without electrical to optical interfaces. Other approaches to photonic integrators based on transversal structures offer high reconfigurability and accuracy owing to the parallel scheme where each path can be controlled independently [194-196]. By tailoring the progressive delay, RF signal integration with a reconfigurable operation bandwidth was achieved [194-196]. Yet these still face limitations arising from the limited number of channels in cases where laser arrays or electro-optical comb sources were employed. These approaches have 
a trade-off between the number of wavelengths and system complexity and ultimately lead to limited channel numbers, and in turn, time-bandwidth products.

Integrated micro-combs [38, 47, 68-70,118,197] offer many advantages for RF integration, including higher numbers of wavelengths and greatly reduced footprint and complexity. Recently, a highly reconfigurable photonic RF temporal integrator was demonstrated with an integrated soliton crystal micro-comb source [123], where the RF signal was imprinted onto the micro-comb lines and progressively delayed via dispersion, and then summed upon detection. The large number of wavelengths (81) enabled a large integration time window of $\sim 6.8 \mathrm{~ns}$ with a time resolution of $\sim 84 \mathrm{ps.}$ The integrator was successfully tested with different input signals, with experimental results matching well with theory.

Figure 19 shows the operation principle of a photonic RF integrator. The integration process can be achieved via a discrete time-spectrum convolution operation between an RF input signal $f(t)$ and flattened micro-comb. With a delay step $\Delta t$, the operation is:

$$
y(t)=\sum_{k=1}^{N} f(t+k \cdot \Delta t)
$$

where $j=\sqrt{-1}, N$ is the total number of wavelengths. After the replicas of $f(t)$ are delayed progressively and summed, the integration of $f(t)$ results, with a time feature [194] of $\Delta t$, an operation bandwidth of $1 / \Delta t$, and a total integration time window of $T=N \times \Delta t$. The frequency-domain transmission response of an ideal non-discrete integrator is linear to $1 /(j \omega)$, where $\omega$ is the angular frequency. The frequency transmission response of the discrete integrator was a low pass sinc filter [111,117], described as

$$
H(\omega)=\sum_{n=0}^{N-1} e^{-j \omega n \Delta t}
$$

The experimental setup of the photonic RF integrator was similar to Fig. 4, based on a soliton crystal micro-comb with a free spectral range of $\sim 49 \mathrm{GHz}$. The soliton crystal micro-comb featured very low intensity noise to the extent that it has even been used as a photonic local oscillator [120]. Thus, the noise of the micro-comb source did not introduce any observable deterioration in the overall noise performance of the integrator. After flattening the microcomb spectral lines with a WaveShaper, the RF signal was imprinted onto the comb lines via an electro-optical modulator (EOM). The replicas were progressively delayed by a spool of standard SMF $(\sim 13 \mathrm{~km})$ and summed upon photodetection using a high-speed photodetector. The delay between adjacent wavelengths $\Delta t$, yielded the resolution of the integrator [194] at $\sim 84 \mathrm{ps,} \mathrm{determined} \mathrm{by} \mathrm{the} \mathrm{dispersion} \mathrm{and} \mathrm{fibre} \mathrm{length} \mathrm{and} \mathrm{spacing} \mathrm{between} \mathrm{the} \mathrm{comb} \mathrm{lines.}$ The operation bandwidth of the integrator was $1 / \Delta t=11.9 \mathrm{GHz}$. The temporal resolution was determined by the delay step of the wavelength channels, and thus in theory can be arbitrarily small by reducing the amount of dispersion, although with a trade-off that the integration window also decreases. Sixty comb lines in the C-band were selected, yielding an integration time window $(T=N \times \Delta t)$ of $60 \times 84 \mathrm{ps}=5.04 \mathrm{~ns}$ (yellow shaded region in Fig. 20). Signal integration for different RF input signals was performed. The red curves in Figs. 20(a)-(c) show the results for Gaussian pulses (blue curves) with an FWHM from $0.20 \mathrm{~ns}$ to $0.94 \mathrm{~ns}$, with the integration window $T$ ( $\sim 5 \mathrm{~ns})$ matching well with theory (5.04 ns). Figures $20(\mathrm{~d})-(\mathrm{e})$ shows the results for dual Gaussian pulses with time intervals of $1.52 \mathrm{~ns}$ and $3.06 \mathrm{~ns}$. The measured results (red curves) clearly illustrate the performance of the integrator by exhibiting three distinct intensity steps in the integration waveforms. The left step corresponds to the integration of the first pulse while the middle step indicates the integration of both the two initial pulses, and the right step shows the integration of only the second pulse since it is beyond the integration window of the first pulse. The integrator was further tested with a rectangular input waveform with a width equal to the integration window (5 ns). The measured integrated waveform exhibited a triangular shape that matched well with theory (gray curve).

Figure 20 shows small residual discrepancies between experiment (red curves) and theory (grey curves). The errors were a result of the non-ideal impulse response of the system, caused by gain variation of the optical amplifier, modulator and photodetector. To verify this, the impulse response of the system was measured with a Gaussian pulse input. Since the time resolution of the system $(\sim 84 \mathrm{ps})$ was much less than the pulse width, the wavelength channels were separated into multiple subsets (each with a much larger spacing between the adjacent comb lines and thus obtained a temporal resolution larger than the input pulse duration), and their impulse responses measured sequentially. Figure 21(a) shows the measured impulse response of the system, which was not flat even when the comb lines were perfectly flattened. The measured impulse response and the input RF signal in Fig. 20 was used to calculate the corresponding integral output, with the results matching the experiment well, showing the errors were induced by the 
non-ideal impulse response of the system.

To reduce these errors, more accurate comb shaping was used, where the error signal of the feedback loop was generated directly by the measured impulse response, instead of the optical power of the comb lines. A flattened impulse response was obtained (Fig. 21(b)), which was much closer to the ideal impulse response than Fig. 21(a). The integration was then performed with the same RF inputs, the results of which are shown in Fig. 22. Note that during this measurement, 81 wavelength channels were enabled by the impulse response shaping process, so that the integration time window $(T=N \times \Delta t)$ increased to $81 \times 84 \mathrm{ps}=6.804 \mathrm{~ns}$, resulting in an operation bandwidth of $1 / 84 \mathrm{ps}$ $=11.9 \mathrm{GHz}$ and a time-bandwidth product of $6.804 \mathrm{~ns} \times 11.9 \mathrm{GHz}=\sim 81$ (approximately equal to the number of channels $N$ ). The measured integrated results (red curves, Fig. 22) show significantly better agreement with theory, indicating the success of the impulse response shaping method as well as the feasibility of this approach to photonic RF integration.

\section{DISCUSSION}

We have reviewed recent progress on the use of Kerr micro-combs for temporal RF and microwave signal processing, focusing on integral and continuously tunable (in order) fractional Hilbert transformers, differentiators, and finally integrators. Micro-combs produce a large number of comb lines that greatly increase the performance and processing bandwidth of RF systems for the amplitude and phase response. Micro-combs have shown their powerful capabilities for RF signal processing due to their large number of comb lines, their compact footprint, and large comb spacings that can be generated compared to other approaches such as electro-optic combs. The devices reached operation bandwidths of $\sim 16 \mathrm{GHz}$, for the $49 \mathrm{GHz}$ devices and $\sim 25 \mathrm{GHz}$ for 200GHz FSR combs, evaluated both in the frequency domain using Vector Network Analyzers and in the time domain with Gaussian pulse input signals.

We have highlighted the advantages of using Kerr micro-combs as the basis for RF photonic microwave transversal filters, illustrating the tradeoffs between using widely spaced micro-combs $(200 \mathrm{GHz})$ and record-low spaced microcombs $(49 \mathrm{GHz})$ in terms of performance. The greater number of lines supplied by the $49 \mathrm{GHz}$ comb (80 versus 20 for the $200-\mathrm{GHz}$ device) yielded significantly better system performance. On the other hand, the $49-\mathrm{GHz}$ device is more limited in operational bandwidth, being restricted to roughly the Nyquist zone of $25 \mathrm{GHz}$. The 200-GHz devices were able to reach RF frequencies well beyond what conventional electronic microwave technology can achieve.

For transversal frequency-domain signal processors such as filters, Hilbert transformers and differentiators, the number of wavelengths determines the number of taps, which in turn directly determines the system performance. Two qualitatively different combs have been employed to realize RF functions - a widely spaced 200GHz FSR microcomb operating in a quasi-coherent state that does not feature solitons, as well as a low spaced microcomb with an FSR of $49 \mathrm{GHz}$, operating in a state referred to as soliton crystals. Within the optical $\mathrm{C}$ band, the $49 \mathrm{GHz}$ spaced microcomb enabled up to 90 comb lines, yielding significantly better performance for the temporal signal processors than the than the $200 \mathrm{GHz}$ spaced microcomb which only allowed 20 taps in the C-band. However, the $49 \mathrm{GHz}$ combs generally yielded smaller Nyquist bandwidths of $\sim 16 \mathrm{GHz}$. For the transversal temporal signal processors such as the integrators, the large number of wavelengths brought about a large number of broadcasted RF replicas, thus yielding a large integration window for the integrator. The temporal response of the integrator was measured with a diverse range of RF inputs, verifying a integration window of $6.8 \mathrm{~ns}$ and a time feature as fast as $84 \mathrm{ps}$. The good agreement with theory and experiment verified that this approach is an effective way to implement high-speed reconfigurable signal processing featuring high processing bandwidths, for future ultra-high-speed microwave systems.

We believe that micro-combs will bring further benefits to RF photonic signal processing in many respects. First, the coherent nature of the soliton states will enable more advanced RF functions such as wideband frequency conversion and clock generation. Maturing nanofabrication techniques have allowed the realization of high-Q MRRs for comb generation with FSRs ranging from 10s' of GHz [119] up to the THz regime [47], covering the full RF bands of interest for almost any integrated RF systems. The wide available FSR spacing of optical micro-combs allows for a large Nyquist zone up to 100's of GHz. This is well beyond the processing bandwidth of electronic devices and is also challenging for mode-locked lasers and OFCs obtained by EO modulation. With tailored dispersion, ultra-wideband bandwidth, up to even octave-spanning microcombs, [71] can be generated, thus enabling a large number of wavelength channels to be achieved with a single on-chip source, to work in conjunction with broadband opto-electronic 
equipment. This approach enables significantly enhanced wavelength-division parallelism for massive data transmission and processing [62-64] for many applications including radio-over-fibre systems, for example.

Further, the high reconfigurability of micro-comb-based programmable RF photonic transversal filters enables versatile processing functions and operation bandwidths for diverse computing requirements of many practical applications without having to alter any hardware. By simply programming and shaping the comb lines according to specific tap coefficients, the same physical equipment can be employed to achieve multiple signal processing functions. Such a high degree of reconfigurability enable highly reconfigurable processing functions and high processing accuracy that typically cannot be obtained by passive photonic integrated circuits or via optical analogue signal processing. Photonic transversal filters are essentially equivalent to filters achieved via purely digital signal processing, but implemented by photonic hardware.

There are a number of factors that lead to tap errors during the comb shaping, thus leading to non-ideal impulse response of the system as well as deviations between the experimental results and theory. These factors mainly include the instability of the optical micro-combs, the accuracy of the waveshapers, the gain variation of the optical amplifier, the chirp induced by the optical modulator, and the third-order dispersion of the dispersive fibre. To combat this, realtime feedback control paths can be employed to reduce the errors. In this approach, the power of the comb lines is first detected by an optical spectrum analyser (OSA) and then compared with the ideal tap weights, generating an error signal that is fed back into the waveshaper to calibrate the system and achieve accurate comb shaping. To further reduce the tap errors and improve the accuracy of comb shaping, the error signal of the feedback loop can be generated directly by the measured impulse response, instead of the optical power of the comb lines. In this approach, replicas of an RF Gaussian pulse are measured at all wavelengths to obtain the impulse response of the system, whose peak intensities are further extracted to obtain accurate RF-to-RF wavelength channel weights. Following this, the extracted channel weights are subtracted from the desired weights to obtain an error signal that is used to program the loss of the Waveshaper. After several iterations of the comb shaping loop, an accurate impulse response that compensates the non-ideal impulse response of the system can be obtained, thus significantly improving accuracy of the RF photonic transversal filter based signal processors.

Finally, RF photonic signal processors based on Kerr micro-combs have significant potential to achieve high levels of integration with current nanofabrication techniques. To begin with, the most challenging component is the microcomb source itself, and this is now able to be fabricated with CMOS compatible processes. Globally established CMOS fabrication foundries can achieve advanced hybrid integration of microcomb sources and III-V devices, and this will potentially enable monolithic integration of the entire RF system. Other key components have been realized in integrated form with state-of-art nanofabrication techniques [198-200], including the optical pump source [131, 139], $\mathrm{LiNO}_{3}$ modulators [200], optical spectral shapers [198], large dispersion media [199] and photodetectors. Further, advanced integrated microcombs have been demonstrated [139] that can generate soliton crystals reliably in turn-key operation. Monolithically integrating the whole processing system would greatly strengthen the performance, compactness and energy efficiency of the system. Even without this, however, using the discrete integrated comb sources to replace discrete laser arrays already yields significant benefits for RF and microwave systems in terms of size, cost, and complexity.

\section{CONCLUSION}

We review recent work on the applications of Kerr micro-combs to photonic RF and microwave temporal signal processing based on transversal filters. Optical micro-combs bring a new generation of compact multi-wavelength sources to the RF photonics community and offer enormous possibilities for achieving high-performance RF signal processing with reduced footprint, lower complexity, and potentially lower cost. By programming and shaping the comb lines according to the calculated tap weights, diverse signal processing functions are designed and experimentally demonstrated. We focus on fractional and integral order Hilbert transforms and differentiators as well as an integrator, based on integrated Kerr micro-comb sources that operates on the RF signal rather than the complex optical field. Realtime system demonstrations are also performed and show good agreement with theory. These results verify that the Kerr micro-comb is an effective way to implement high-speed reconfigurable signal processing featuring high processing bandwidths and reconfigurability for future ultra-high-speed RF systems 


\section{References}

[1] S. Radic, and D. J. Moss, and B. J. Eggleton, "Nonlinear optics in communications: From crippling impairment to ultrafast tools", Chapter 20, p759-828 in Optical Fiber Telecommunications V: Components and Sub-systems, Ed. Ivan P. Kaminow, Tingye Li, and Alan E. Willner, Academic Press, Oxford, UK, February 2008.

[2] J. Leuthold, C. Koos, and W. Freude, "Nonlinear silicon photonics," Nat. Photon., vol. 4, no. 8, pp. 535-544, 2010.

[3] L. Li, P. G. Patki, Y.B. Kwon, et al. "All-optical regenerator of multi-channel signals," Nature Communications, vol. 8, Article number: 884, 2017.

[4] F. Li, et al., "All-optical XOR logic gate for 40Gb/s DPSK signals via FWM in a silicon nanowire," Optics Express, vol. 19, no. 21, pp. 20364-20371, $2011 . \quad$ DOI: 10.1364/OE.19.020364.

[5] F. Li, et al., "Error-free All-Optical Demultiplexing at 160Gb/s via FWM in a Silicon Nanowire," Optics Express, vol. 18, no. 4, pp. 3905-3910, 2010.

DOI: 10.1364/OE.18.003905.

[6] H. Ji, et al., "1.28-Tb/s Demultiplexing of an OTDM DPSK Data Signal Using a Silicon Waveguide," Photonics Technology Letters, vol. 22, no. 23, pp. 1762-1764, 2010.

[7] C. Monat, et al., "Investigation of phase matching for third-harmonic generation in silicon slow light photonic crystal waveguides using Fourier optics," Optics Express, vol. 18, no. 7, pp. 6831-6840, 2010. DOI: 10.1364/OE.18.006831.

[8] B. Corcoran, et al., "Optical Signal Processing on a Silicon Chip at 640Gb/s Using Slow-Light," Optics Express, vol. 18, no. 8, pp. 7770-7781, 2010. DOI: 10.1364/OE.18.007770.

[9] V. G. Ta'eed, et al., "Integrated all-optical pulse regenerator in chalcogenide waveguides," Optics Letters, vol. 30, no. 21, pp. 2900-2902, 2005. DOI: 10.1364/OL.30.002900.

[10] M. Rochette, et al., "Bit-error-ratio improvement with 2R optical regenerators," IEEE Photonics Technology Letters, vol. 17, no. 4, pp. 908-910, 2005.

[11] M. Ferrera, et al., "CMOS compatible integrated all-optical radio frequency spectrum analyzer," Optics Express, vol. 22, no. 18, pp. 21488 - 21498, 2014. DOI: 10.1364/OE.22.021488.

[12] C. Monat, et al., "Integrated optical auto-correlator based on third-harmonic generation in a silicon photonic crystal waveguide," Nature Communications, vol. 5, Article:3246, 2014. doi:10.1038/ncomms4246.

[13] F. Li, et al., "All-optical wavelength conversion for $10 \mathrm{~Gb} / \mathrm{s}$ DPSK signals in a silicon ring resonator," Optics Express, vol. 19, no. 23, pp. 22410-22416, 2011.

[14] T. D. Vo, et al., "Silicon-Chip-Based Real-Time Dispersion Monitoring for 640 Gbit/s DPSK Signals," IEEE Journal of Lightwave Technology, vol. 29, no. 12, pp. 1790-1796, 2011.

[15] M. Ferrera, et al., "All-optical 1 $1^{\text {st }}$ and $2^{\text {nd }}$ order integration on a chip," Optics Express, vol. 19, no. 23, pp. 23153-23161, 2011.

[16] B. Corcoran, et al., "Silicon nanowire based radio-frequency spectrum analyzer," Optics Express, vol. 18, no. 19, pp. 2019020200, 2010. DOI: 10.1364/OE.18.020190.

[17] B. Corcoran, et al., "Green light emission in silicon through slow-light enhanced third-harmonic generation in photonic-crystal waveguides," Nature Photonics, vol. 3, no. 4, pp. 206-210, 2009. doi:10.1038/nphoton.2009.28.

[18] D. J. Moss, H. M. van Driel, and J. E. Sipe, "Dispersion in the anisotropy of optical third-harmonic generation in silicon," Opt. Lett., vol. 14, no. 1, pp. 57-59, 1989.

[19] J. E. Sipe, D. J. Moss, and H. M. van Driel, "Phenomenological Theory of Optical Second- And Third-Harmonic Generation Form Cubic Centrosymmetric Crystals," Phys. Rev. B, vol. 35, no. 3, pp. 1129-1141, 1987.

[20] D. J. Moss, E. Ghahramani, J. E. Sipe, and H. M. van Driel, "Band-structure calculation of dispersion and anisotropy in $\chi \rightarrow(3)$ for third-harmonic generation in Si, Ge, and GaAs," Phys. Rev. B, vol. 41, no. 3, pp. 1542-1560, 1990.

[21] D. J. Moss, H. M. van Driel, and J. E. Sipe, "Third harmonic generation as a structural diagnostic of ion implanted amorphous and crystalline silicon,” Appl. Phy. Lett., vol. 48, no. 17, pp. 1150, 1986.

[22] D. J. Moss, et al., "Ultrafast all-optical modulation via two-photon absorption in silicon-insulator waveguides," Electronics Letters, vol. 41, no. 6, pp. 320-321, 2005. DOI:10.1049/el:20058051

[23] M. R. E. Lamont, et al.,"Two-photon absorption effects on self-phase-modulation-based 2R optical regeneration," Photonics Technology Letters, vol. 18, no. 10, pp. 1185-1187, 2006. DOI:10.1109/LPT.2006.874718.

[24] A.Tuniz, G. Brawley, D. J. Moss, and B. J. Eggleton, "Two-photon absorption effects on Raman gain in single mode As2Se3 chalcogenide glass fiber," Optics Express, vol. 16, no. 22, pp. 18524-18534, 2008. DOI: 10.1364/OE.16.018524.

[25] B. J. Eggleton, B. Luther-Davies, and K. Richardson, "Chalcogenide photonics", Nature Photonics, vol. 5, no. 3, pp. 141-148, 2011.

[26] M. Lee, et al., "Photosensitive post tuning of chalcogenide photonic crystal waveguides," Optics Express, vol. 15, no. 3, pp. 12771285, 2007. DOI:10.1364/OE.15.001277

[27] S. Tomljenovic-Hanic, M. J. Steel, C. M. d. Sterke and D. J. Moss, "High-Q cavities in photosensitive photonic crystals", Optics Letters, 32, no. 5, pp. 542-544, 2007.

[28] C. Grillet, et al., "Nanowire coupling to photonic crystal nanocavities for single photon sources," Optics Express, vol. 15, no. 3, pp. 1267-1276, 2007. DOI:10.1364/OE.15.001267

[29] V. G. Ta'eed, et al., "Ultrafast all-optical chalcogenide glass photonic circuits", Optics Express, vol. 15, no. 15, pp. 9205-9221, 2007. 
[30] D. Freeman, et al., "Chalcogenide Glass Photonic Crystal Devices", Photonic and Electromagnetic Crystal Structures, Photonics and Nanostructures-Fundamentals and Applications, Science Direct Elsevier Publishing, vol. 6, no. 1, pp. 3-11, 2008. doi:10.1016/j.photonics.2007.11.001.

[31] C. Grillet, et al., "Characterization and modeling of Fano resonances in chalcogenide photonic crystal membranes", Optics Express, vol. 14, no. 1, pp. 369-376, 2006.

[32] V. G. Ta'eed, et al., "Self-phase modulation based integrated optical regeneration in chalcogenide waveguides", IEEE Journal of Selected Topics in Quantum Electronics, vol. 12, no. 3, pp. 360-370, 2006.

[33] M. Shokooh-Saremi, et al., "High performance Bragg gratings in chalcogenide rib waveguides written with a modified Sagnac interferometer: experiment and modeling", Journal of the Optical Society of America B (JOSA B), vol. 23, no. 7, pp. 1323-1331, 2006.

[34] M. R. E. Lamont, et al., "Error-free wavelength conversion via cross phase modulation in $5 \mathrm{~cm}$ of As2S3 chalcogenide glass rib waveguide", Electronics Letters, vol. 43, pp. 945-947, 2007.

[35] K. Ikeda, R. E. Saperstein, N. Alic, and Y. Fainman, "Thermal and Kerr nonlinear properties of plasma-deposited silicon nitride/silicon dioxide waveguides", Opt. Express, vol. 16, pp. 12987-12994, 2008.

[36] J. S. Levy, A. Gondarenko, M. A. Foster, et al., "CMOS-compatible multiple-wavelength oscillator for on-chip optical interconnects," Nature Photonics, vol. 4, 1, pp. 37-40, 2010.

[37] L. Razzari, D. Duchesne, M. Ferrera, et al., "CMOS-compatible integrated optical hyper-parametric oscillator," Nature Photonics, vol. 4, no. 1, pp. 41-45, 2010.

[38] D. J. Moss, R. Morandotti, A. L. Gaeta, et al., "New CMOS-compatible platforms based on silicon nitride and Hydex for nonlinear optics," Nature Photonics, vol. 7, no. 8, pp. 597-607, 2013.

[39] M. Ferrera, L. Razzari, D. Duchesne, et al., "Low-power continuous-wave nonlinear optics in doped silica glass integrated waveguide structures," Nature Photonics, vol. 2, no. 12, pp. 737-740, 2008.

[40] M. Ferrera, D. Duchesne, L. Razzari, et al., "Low power four wave mixing in an integrated, micro-ring resonator with $\mathrm{Q}=1.2$ million," Optics Express, vol. 17, no. 16, pp. 14098-14103, 2009.

[41] D. Duchesne, M. Peccianti, M. R. E. Lamont, et al., "Supercontinuum generation in a high index doped silica glass spiral waveguide," Optics Express, vol. 18, no, 2, pp. 923-930, 2010.

[42] M. Ferrera, et al., "On-chip CMOS-compatible all-optical integrator", Nature Communications, vol. 1, Article $29,2010$. DOI: $10.1038 /$ ncomms 1028

[43] A. Pasquazi, et al., "All-optical wavelength conversion in an integrated ring resonator," Optics Express, vol. 18, no. 4, pp. 38583863, 2010.

[44] A. Pasquazi, Y. Park, J. Azana, et al., "Efficient wavelength conversion and net parametric gain via Four Wave Mixing in a high index doped silica waveguide," Optics Express, vol. 18, no. 8, pp. 7634-7641, 2010.

[45] M. Peccianti, M. Ferrera, L. Razzari, et al., "Subpicosecond optical pulse compression via an integrated nonlinear chirper," Optics Express, vol. 18, no. 8, pp. 7625-7633, 2010.

[46] D. Duchesne, M. Ferrera, L. Razzari, et al., "Efficient self-phase modulation in low loss, high index doped silica glass integrated waveguides," Optics Express, vol. 17, no. 3, pp. 1865-1870, 2009.

[47] A. Pasquazi, M. Peccianti, L. Razzari, D. J. Moss, S. Coen, M. Erkintalo, Y. K. Chembo, T. Hansson, S. Wabnitz, P. Del'Haye, X. X. Xue, A. M. Weiner, and R. Morandotti, "Micro-combs: A novel generation of optical sources," Physics Reports, vol. 729, pp. 1-81, Jan 27. 2018.

[48] P. Del'Haye, A. Schliesser, O. Arcizet, T. Wilken, R. Holzwarth, and T. J. Kippenberg, "Optical frequency comb generation from a monolithic microresonator", Nature, vol. 450, pp. 1214-1217, 2007.

[49] M. Peccianti, et al., "Demonstration of an ultrafast nonlinear microcavity modelocked laser", Nature Communications, vol. 3, pp. 765, 2012. DOI: $10.1038 /$ ncomms 1762

[50] M. Kues, et al., "Passively modelocked laser with an ultra-narrow spectral width", Nature Photonics, vol. 11, no. 3, pp. $159,2017$. DOI:10.1038/nphoton.2016.271

[51] A. Pasquazi, L. Caspani, M. Peccianti, et al., "Self-locked optical parametric oscillation in a CMOS compatible microring resonator: a route to robust optical frequency comb generation on a chip," Optics Express, vol. 21, no. 11, pp. 13333-13341, 2013.

[52] A. Pasquazi, M. Peccianti, B. E. Little, et al., "Stable, dual mode, high repetition rate mode-locked laser based on a microring resonator," Optics Express, vol. 20, no. 24, pp. 27355-27362, 2012.

[53] C. Reimer, L. Caspani, M. Clerici, et al., "Integrated frequency comb source of heralded single photons," Optics Express, vol. 22, no. 6, pp. 6535-6546, 2014.

[54] C. Reimer, et al., "Cross-polarized photon-pair generation and bi-chromatically pumped optical parametric oscillation on a chip", Nature Communications, vol. 6, Article 8236, 2015. DOI: 10.1038/ncomms9236

[55] L. Caspani, C. Reimer, M. Kues, et al., "Multifrequency sources of quantum correlated photon pairs on-chip: a path toward integrated Quantum Frequency Combs," Nanophotonics, vol. 5, no. 2, pp. 351-362, 2016.

[56] C. Reimer, M. Kues, P. Roztocki, B. Wetzel, F. Grazioso, B. E. Little, S. T. Chu, T. Johnston, Y. Bromberg, L. Caspani, D. J. Moss, and R. Morandotti, "Generation of multiphoton entangled quantum states by means of integrated frequency combs," Science, vol. 351, no. 6278, pp. 1176-1180, 2016.

[57] M. Kues, et al., "On-chip generation of high-dimensional entangled quantum states and their coherent control", Nature, vol. 546, no. 7660, pp. 622-626, 2017. 
[58] P. Roztocki, M. Kues, C. Reimer, B. Wetzel, S. Sciara, Y. Zhang, A. Cino, B. E. Little, S. T. Chu, D. J. Moss, and R. Morandotti, "Practical system for the generation of pulsed quantum frequency combs," Optics Express, vol. 25, no. 16, pp. 18940-18949, 2017.

[59] Y. Zhang, et al., "Induced photon correlations through superposition of two four-wave mixing processes in integrated cavities", Laser and Photonics Reviews, vol. 14, no. 7, pp. 2000128, 2020. DOI: 10.1002/lpor.202000128

[60] M. Kues, C. Reimer, A. Weiner, J. Lukens, W. Munro, D. J. Moss, and R. Morandotti, "Quantum Optical Micro-combs", Nature Photonics, vol. 13, no.3, pp. 170-179, 2019.

[61] C. Reimer, et al.,"High-dimensional one-way quantum processing implemented on d-level cluster states", Nature Physics, vol. 15, no.2, pp. 148-153, 2019.

[62] P. Marin-Palomo, et al., "Microresonator-based solitons for massively parallel coherent optical communications", Nature, vol. 546, no. 7657, pp. 274, 2017.

[63] J. Pfeifle, V. Brasch, M. Lauermann, Y. Yu, D. Wegner, T. Herr, K. Hartinger, et al., "Coherent terabit communications with microresonator Kerr frequency combs", Nature Photonics, vol. 8, no. 5, pp. 375-380, 2014.

[64] B. Corcoran, et al., "Ultra-dense optical data transmission over standard fiber with a single chip source", Nature Communications, vol. 11, Article:2568, 2020. DOI:10.1038/s41467-020-16265-x.

[65] X. Xu, et al., "Photonic perceptron based on a Kerr microcomb for scalable high speed optical neural networks", Laser and Photonics Reviews, vol. 14, no. 8, 2020. DOI:10.1002/lpor.202000070.

[66] X. Xu, et al., "11 TeraFLOPs photonic convolutional accelerator for deep learning convolutional optical neural networks", submitted 2020.

[67] J. Feldmann, et al., "Parallel convolution processing using an integrated photonic tensor core", arXiv preprint arXiv:2002.00281, 2020.

[68] D. T. Spencer, et al., "An optical-frequency synthesizer using integrated photonics", Nature, vol. 557, no. 7703, pp. 81-85, 2018.

[69] T. J. Kippenberg, A. L. Gaeta, M. Lipson, and M. L. Gorodetsky, "Dissipative Kerr solitons in optical microresonators," Science, vol. 361, no. 6402, 2018.

[70] A. L. Gaeta, M. Lipson, and T. J. Kippenberg, "Photonic-chip-based frequency combs," Nature Photonics, vol. 13, no. 3, pp. 158169, Mar. 2019.

[71] P. Del'Haye, T. Herr, E. Gavartin, M. L. Gorodetsky, R. Holzwarth, and T. J. Kippenberg, "Octave spanning tunable frequency comb from a microresonator", Physical Review Letters, vol. 107, no. 6, pp. 063901, 2011.

[72] T. J. Kippenberg, R. Holzwarth, S. A. Diddams, "Microresonator-based optical frequency combs", Science, vol. 332, no. 6029, pp. 555-559, 2011.

[73] T. Herr, V. Brasch, J. D. Jost, C. Y. Wang, N. M. Kondratiev, M. L. Gorodetsky, et al., "Temporal solitons in optical microresonators", Nature Photonics, vol. 8, no. 2, pp. 145-152, 2014.

[74] F. Ferdous, H. Miao, D. E. Leaird, K. Srinivasan, J. Wang, L. Chen, L. T. Varghese, and A. M. Weiner, "Spectral line-by-line pulse shaping of on-chip microresonator frequency combs", Nature Photonics, vol. 5, no. 12, pp. 770, 2011.

[75] X. Xue, P. H. Wang, Y. Xuan, M. Qi, and A. M. Weiner, "Microresonator Kerr frequency combs with high conversion efficiency", Laser \& Photonics Reviews, vol. 11, no. 1, 2017.

[76] X. Xue, M. Qi, and A. M. Weiner, "Normal-dispersion microresonator Kerr frequency combs", Nanophotonics, vol. 5, no. 2, pp. 244-262, 2016.

[77] C. Grillet, et al., "Amorphous Silicon Nanowires with Record High Nonlinearity, FOM, and Optical Stability", Optics Express, vol. 20, no. 20. pp. 22609-22615, 2012.

[78] J. W. Choi, B. Sohn, G. F. R. Chen, D. K. T. Ng, and D. T. H. Tan, "Soliton-effect optical pulse compression in CMOS-compatible ultra-silicon-rich nitride waveguides," APL Photonics, HIBSP2019, pp. 110804, 2020.

[79] J. Capmany, and D. Novak, "Microwave photonics combines two worlds," Nat. Photonics, vol. 1, no. 6, pp. 319-330, 2007.

[80] J. P. Yao, "Microwave photonics," Journal of Lightwave Technol., vol. 27, no. 1-4, pp. 314-335, Jan-Feb. 2009.

[81] D. Marpaung, J. Yao, and J. Capmany, "Integrated microwave photonics," Nature Photonics, vol. 13, no. 2, pp. 80-90, Feb. 2019.

[82] J. Azaña, "Ultrafast analog all-optical signal processors based on fiber-grating devices," IEEE Photonics Journal, vol. 2, no. 3, pp. 359-386. 2010.

[83] J. Capmany, B. Ortega, and D. Pastor, “A tutorial on microwave photonic filters," Journal of Lightwave Technol., vol. 24, no. 1, pp. 201-229, 2006.

[84] V. R. Supradeepa et al., "Comb-based radiofrequency photonic filters with rapid tunability and high selectivity," Nat. Photonics, vol. 6, no. 3, pp. 186-194, Mar.2012.

[85] J. Wu, X. Xu, T. G. Nguyen, S. T. Chu, B. E. Little, R. Morandotti, A. Mitchell, and D. J. Moss, "RF Photonics: An Optical Microcombs' Perspective," IEEE J. Sel. Top. Quantum Electron., vol. 24, no. 4, pp. 6101020, Jul-Aug. 2018. DOI: 10.1109/JSTQE.2018.2805814.

[86] V. Torres-Company, and A. M. Weiner, "Optical frequency comb technology for ultra-broadband radio-frequency photonics," Laser Photonics Rev, vol. 8, no. 3, pp. 368-393, May. 2014.

[87] Z. Jiang, C. B. Huang, D. E. Leaird, and A. M. Weiner, "Optical arbitrary waveform processing of more than 100 spectral comb lines," Nat Photonics, vol. 1, no. 8, pp. 463-467, Aug. 2007.

[88] Y. Liu, J. Hotten, A. Choudhary, B. J. Eggleton, and D. Marpaung, "All-optimized integrated RF photonic notch filter," Opt. Lett., vol. 42, no. 22, pp. 4631-4634, Nov 15. 2017. 
[89] Y. Liu, D. Marpaung, A. Choudhary, J. Hotten, and B. J. Eggleton, "Link performance optimization of chip-based Si3N4 microwave photonic filters," J. Lightwave Technol., vol. 36, no. 19, pp. 4361-4370, 2018.

[90] Y. Liu, Y. Yu, S. X. Yuan, X. B. Xu, and X. L. Zhang, "Tunable megahertz bandwidth microwave photonic notch filter based on a silica microsphere cavity," Opt. Lett., vol. 41, no. 21, pp. 5078-5081, Nov 1. 2016.

[91] D. Marpaung, B. Morrison, M. Pagani, R. Pant, D. Y. Choi, B. Luther-Davies, S. J. Madden, and B. J. Eggleton, "Low-power, chip-based stimulated Brillouin scattering microwave photonic filter with ultrahigh selectivity," Optica, vol. 2, no. 2, pp. 76-83, Feb 20. 2015.

[92] A. Choudhary, B. Morrison, I. Aryanfar, S. Shahnia, M. Pagani, Y. Liu, K. Vu, S. Madden, D. Marpaung, and B. J. Eggleton, "Advanced integrated microwave signal processing with giant on-chip Brillouin gain," J. Lightwave Technol., vol. 35, no. 4, pp. 846-854, Feb 15. 2017.

[93] D. Marpaung, B. Morrison, R. Pant, and B. J. Eggleton, "Frequency agile microwave photonic notch filter with anomalously high stopband rejection," Opt. Lett., vol. 38, no. 21, pp. 4300-4303, Nov 1. 2013.

[94] X. Q. Zhu, F. Y. Chen, H. F. Peng, and Z. Y. Chen, "Novel programmable microwave photonic filter with arbitrary filtering shape and linear phase," Opt. Express, vol. 25, no. 8, pp. 9232-9243, Apr 17. 2017.

[95] F. Jiang, Y. Yu, H. T. Tang, L. Xu, and X. L. Zhang, "Tunable bandpass microwave photonic filter with ultrahigh stopband attenuation and skirt selectivity," Opt. Express, vol. 24, no. 16, pp. 18655-18663, Aug 8. 2016.

[96] Z. J. Zhu, H. Chi, T. Jin, S. L. Zheng, X. F. Jin, and X. M. Zhang, "All-positive-coefficient microwave photonic filter with rectangular response," Opt. Lett., vol. 42, no. 15, pp. 3012-3015, Aug 1. 2017.

[97] G. Yu, W. Zhang, and J. A. R. Williams, "High-performance microwave transversal filter using fiber Bragg grating arrays," IEEE Photonic Tech L, vol. 12, no. 9, pp. 1183-1185, Sep. 2000.

[98] J. S. Leng, W. Zhang, and J. A. R. Williams, "Optimization of superstructured fiber Bragg gratings for microwave photonic filters response," IEEE Photonic Tech L, vol. 16, no. 7, pp. 1736-1738, Jul. 2004.

[99] D. B. Hunter, R. A. Minasian, and P. A. Krug, "Tunable optical transversal filter based on chirped gratings," Electron. Lett., vol. 31, no. 25, pp.2205-2207, Dec 7. 1995.

[100] E. Hamidi, D. E. Leaird, and A. M. Weiner, "Tunable Programmable Microwave Photonic Filters Based on an Optical Frequency Comb," IEEE Journal of Microwave Theory, vol. 58, no. 11, pp. 3269-3278, Nov. 2010.

[101] R. Wu, V. R. Supradeepa, C. M. Long, D. E. Leaird, and A. M. Weiner, "Generation of very flat optical frequency combs from continuous-wave lasers using cascaded intensity and phase modulators driven by tailored radio frequency waveforms," Opt. Lett., vol. 35, no. 19, pp. 3234-3236, Oct 1. 2010.

[102] S. Mansoori, and A. Mitchell, "RF transversal filter using an AOTF," IEEE Photonic Tech L, vol. 16, no. 3, pp. 879-881, Mar. 2004.

[103] M. Delgado-Pinar, J. Mora, A. Diez, M. V. Andres, B. Ortega, and J. Capmany, "Tunable and reconfigurable microwave filter by use of a Bragg-grating-based acousto-optic superlattice modulator," Opt. Lett., vol. 30, no. 1, pp. 8-10, Jan 1. 2005.

[104] W. Z. Li, and J. P. Yao, "Optical frequency comb generation based on repeated frequency shifting using two Mach-Zehnder modulators and an asymmetric Mach-Zehnder interferometer," Opt. Express, vol. 17, no. 26, pp. 23712-23718, Dec 21. 2009.

[105] C. H. Chen, C. He, D. Zhu, R. H. Guo, F. Z. Zhang, and S. L. Pan, "Generation of a flat optical frequency comb based on a cascaded polarization modulator and phase modulator," Opt. Lett., vol. 38, no. 16, pp. 3137-3139, Aug 15. 2013.

[106] T. Saitoh, M. Kourogi, and M. Ohtsu, "An optical frequency synthesizer using a waveguide-type optical frequency comb generator at 1.5-mu m wavelength," IEEE Photonic Tech L, vol. 8, no. 11, pp. 1543-1545, Nov. 1996.

[107] T. G. Nguyen et al., "Integrated frequency comb source-based Hilbert transformer for wideband microwave photonic phase analysis," Opt. Express, vol. 23, no. 17, pp. 22087-22097, Aug. 2015.

[108] X. Xue, et al., "Programmable single-bandpass photonic RF filter based on a Kerr comb from a microring," Journal of Lightwave Technol., vol. 32, no. 20, pp. 3557-3565, Oct. 2014.

[109] X. Xu, J. Wu, M. Shoeiby, T. G. Nguyen, S. T. Chu, B. E. Little, R. Morandotti, A. Mitchell, and D. J. Moss, "Reconfigurable broadband microwave photonic intensity differentiator based on an integrated optical frequency comb source," APL Photonics, vol. 2, no. 9, 096104, Sep. 2017.

[110] X. Xu, M. Tan, J. Wu, R. Morandotti, A. Mitchell, and D. J. Moss, "Microcomb-based photonic RF signal processing", IEEE Photonics Technology Letters, vol. 31 no. 23 1854-1857, 2019.

[111] X. Xu, et al., "Advanced RF and microwave functions based on an integrated optical frequency comb source," Opt. Express, vol. 26, no. 3, pp. 2569-2583, Feb. 2018.

[112] X. Xue, et al., "Microcomb-based true-time-delay network for microwave beamforming with arbitrary beam pattern control," Journal of Lightwave Technology, vol. 36, no. 12, pp. 2312-2321, Jun. 2018.

[113] X. Xu, et al., "Broadband RF channelizer based on an integrated optical frequency Kerr comb source," Journal of Lightwave Technology, vol. 36, no. 19, pp. 7, 2018.

[114] X. Xu, et al., "Continuously tunable orthogonally polarized RF optical single sideband generator based on micro-ring resonators," Journal of Optics, vol. 20, no. 11, pp. 115701. 2018.

[115] X. Xu, et al., "Orthogonally polarized RF optical single sideband generation and dual-channel equalization based on an integrated microring resonator," Journal of Lightwave Technology, vol. 36, no. 20, pp. 4808-4818. 2018.

[116] X. Xu, et al., "Photonic microwave true time delays for phased array antennas using a $49 \mathrm{GHz}$ FSR integrated optical micro-comb source," Photonics Res, vol. 6, no. 5, pp. B30-B36, 2018. 
[117] X. Xu, et al., "Advanced adaptive photonic RF filters with 80 taps based on an integrated optical micro-comb source," Journal of Lightwave Technology, vol. 37, no. 4, pp. 1288-1295, 2019.

[118] W. Liang, et al., "High spectral purity Kerr frequency comb radio frequency photonic oscillator," Nature Communications, vol. 6 pp. 7957. 2015.

[119] J. Liu, et al., "Photonic microwave generation in the X-and K-band using integrated soliton microcombs" Nature Photonics, vol. 14 , pp. 1-6, 2020.

[120] X.. Xu, et al., Broadband microwave frequency conversion based on an integrated optical micro-comb source", Journal of Lightwave Technology, vol. 38 no. 2, pp. 332-338, 2020.

[121] M. Tan, et al., "Photonic RF and microwave filters based on 49GHz and 200GHz Kerr microcombs", Optics Comm. vol. 465, Article: 125563, Feb. 22. 2020.

[122] X. Xu, et al., "Broadband photonic RF channelizer with 90 channels based on a soliton crystal microcomb", Journal of Lightwave Technology, Early Access, 2020. doi: 10.1109/JLT.2020.2997699

[123] X. Xu, et al., "Photonic RF and microwave integrator with soliton crystal microcombs", IEEE Transactions on Circuits and Systems II: Express Briefs, Early Access, 2020. DOI:10.1109/TCSII.2020.2995682

[124] X. Xu, et al., "Photonic RF phase-encoded signal generation with a microcomb source", IEEE Journal of Lightwave Technology, vol. 38, no. 7, pp. 1722-1727, 2020.

[125] X. Xu, et al., "High performance RF filters via bandwidth scaling with Kerr micro-combs," APL Photonics, vol. 4, no. 2, pp. 026102. 2019.

[126] M. Tan, et al., "Microwave and RF photonic fractional Hilbert transformer based on a $50 \mathrm{GHz}$ Kerr micro-comb", Journal of Lightwave Technology, vol. 37, no. 24, pp. $6097-6104,2019$.

[127] M. Tan, et al., "RF and microwave fractional differentiator based on photonics", IEEE Transactions on Circuits and Systems: Express Briefs, Early Access, 2020. DOI:10.1109/TCSII.2020.2965158.

[128] M. Tan, et al., "Photonic RF arbitrary waveform generator based on a soliton crystal micro-comb source", submitted, J. Lightwave Technology, 2020.

[129] D. C. Cole, E. S. Lamb, P. Del'Haye, S. A. Diddams, and S. B. Papp, "Soliton crystals in Kerr resonators," Nat. Photonics, vol. 11, no. 10, pp. 671-676, Oct. 2017.

[130] W. Wang, Z. Lu, W. Zhang, S. T. Chu, B. E. Little, L. Wang, X. Xie, M. Liu, Q. Yang, L. Wang, J. Zhao, G. Wang, Q. Sun, Y. Liu, Y. Wang, and W. Zhao, "Robust soliton crystals in a thermally controlled microresonator," Opt. Lett., vol. 43, no. 9, pp. 20022005, 2018.

[131] B. Stern, X. Ji, Y. Okawachi, A. L. Gaeta, and M. Lipson, "Battery-operated integrated frequency comb generator", Nature, vol. 562, no. 7727, pp. 401, 2018.

[132] X. Xue, et al., "Mode-locked dark pulse Kerr combs in normal-dispersion microresonators," Nature Photonics, vol. 9, no. 9, pp. 594. 2015.

[133] H. Bao, et al., "Laser cavity-soliton microcombs," Nature Photonics, vol. 13, no. 6, pp. 384-389, Jun. 2019.

[134] X. Xue, X. Zheng, and B. Zhou, "Super-efficient temporal solitons in mutually coupled optical cavities," Nature Photonics, May 2019.

[135] H. Zhou, et al., "Soliton bursts and deterministic dissipative Kerr soliton generation in auxiliary-assisted microcavities," Light: Science \& Applications, vol. 8, no. 1, pp. 50, 2019.

[136] H. Bao, L. Olivieri, M. Rowley, S. T. Chu, B. E. Little, R. Morandotti, D. J. Moss, J. S. T. Gongora, M. Peccianti and A. Pasquazi, "Turing patterns in a fibre laser with a nested micro-resonator: robust and controllable micro-comb generation", Physical Review Research, vol. 2, pp. 023395, 2020.

[137] L. D. Lauro, J. Li, D. J. Moss, R. Morandotti, S. T. Chu, M. Peccianti, and A. Pasquazi, "Parametric control of thermal selfpulsation in micro-cavities," Opt. Lett. vol. 42, no. 17, pp. 3407-3410, Aug. 2017.

[138] H. Bao, et al., "Type-II micro-comb generation in a filter-driven four wave mixing laser," Photonics Research, vol. 6, no. 5, pp. B67-B73, May 2018.

[139] B. Shen, Chang, L., Liu, J., et al., "Integrated turnkey soliton microcombs," Nature, vol. 582, pp. 365-369, 2020.

[140] D. J. Moss, M. Lamont, S. Mclaughlin, G. Randall, P. Colbourne, S. Kiran and C. A. Hulse,’Tunable dispersion and dispersion slope compensators for 10Gb/s using all-pass multicavity etalons", IEEE Photonics Technology Letters, vol. 15, pp. 730, 2003.

[141] L. M. Lunardi, D. Moss, S. Chandrasekhar, L. L. Buhl, A. Hulse, P. Colbourne, G. Randall, S. Mclaughlin, "Tunable dispersion compensators based on multi-cavity all-pass etalons for 40Gb/s systems", IEEE Journal of Lightwave Technology, vol. 20, pp. 2136, 2002.

[142] D. J. Moss, et al., "Multichannel tunable dispersion compensation using all-pass multicavity etalons", paper TuT2 Optical Fiber Communications Conference, Anaheim (2002). Postconference Technical Digest (IEEE Cat. No.02CH37339). Opt Soc. America. Part vol.1, 2002, pp. 132-3. Washington, DC, USA.

[143] D. J. Moss, et al., "Tunable dispersion compensation at $10 \mathrm{~Gb} / \mathrm{s}$ and $40 \mathrm{~Gb} / \mathrm{s}$ using multicavity all-pass etalons", Optical Fiber Communications Conference (OFC) paper TuD1, page 162, Atlanta, GA, March (2003). Postconference Digest (IEEE Cat. No.03CH37403). Opt. Soc. America. Part vol.1, 2003, pp. 162-3. Washington, DC, USA.

[144] S. L.Hahn, Transforms and Applications Handbook, A. D. Poularikas, Ed., 3rd ed. Boca Raton, FL: CRC Press, 2010 , ch. 7.

[145] L. Moura, "Radio Frequency Implementation of the Fractional Hilbert Transform with Transversal Filters," Circuits, Systems \& Signal Processing., vol. 26, no. 3, pp. 407-417, Jun. 2007. 
[146] A. W. Lohmann, D. Mendlovic, and Z. Zalevsky, "Fractional Hilbert transform," Opt. Lett., vol. 21, no. 4, pp. 281-283, Feb. 1996.

[147] J. A. Davis, D. E. McNamara, and D. M. Cottrell, "Analysis of the fractional Hilbert transform," Appl. Opt., vol. 37, no. 29, pp. 6911-6913, Oct. 1998.

[148] C. D. Holdenried, J. W. Haslett, and B. Davies, "A fully integrated 10-Gb/s tapped delay Hilbert transformer for optical single sideband," IEEE Microw. Wireless Compon. Lett., vol. 15, no. 5, pp. 303-305, May 2005.

[149] H. Emami, N. Sarkhosh, L. A. Bui, and A. Mitchell, "Wideband RF photonic in-phase and quadrature-phase generation," Opt. Lett., vol. 33, no. 2, pp. 98-100, Jan 15. 2008.

[150] M. Li, and J. P. Yao, "All-fiber temporal photonic fractional Hilbert transformer based on a directly designed fiber Bragg grating," Opt. Lett., vol. 35, no. 2, pp. 223-225, Jan. 2010.

[151] M. Li, and J. P. Yao, "Experimental Demonstration of a Wideband Photonic Temporal Hilbert Transformer Based on a Single Fiber Bragg Grating," IEEE Photon. Technol. Lett., vol. 22, no. 21 pp. 1559-1561, Nov. 2010.

[152] M. H. Asghari, and J. Azana, "All-optical Hilbert transformer based on a single phase-shifted fiber Bragg grating: design and analysis," Opt. Lett., vol. 34, no. 3, pp. 334-336, Feb. 2009.

[153] T. Yang, J. Dong, L. Liu, S. Liao, S. Tan, L. Shi, D. Gao, and X. Zhang, "Experimental observation of optical differentiation and optical Hilbert transformation using a single SOI microdisk chip," Sci. Rep., vol. 4, pp. 3960, 2014.

[154] W. Liu, et al., "A Fully Reconfigurable Photonic Integrated Signal Processor," Nature Photonics, vol. 10, no. 3, pp. 190-196, 2016.

[155] Z. Zhang, et al., "Wideband and continuously-tunable fractional photonic Hilbert transformer based on a single high-birefringence planar Bragg grating," Optics Express, vol. 26, pp. 20450-20458, 2018.

[156] F. Zeng, and J. Yao, "An Approach to Ultrawideband Pulse Generation and Distribution Over Optical Fiber," IEEE Photonics Technol. Lett., vol. 18, no. 7, pp. 823-825, Apr. 2006.

[157] S. Pan, and J. Yao, "Optical generation of polarity- and shape-switchable ultrawideband pulses using a chirped intensity modulator and a first-order asymmetric Mach-Zehnder interferometer," Opt. Lett., vol. 34, no. 9, pp. 1312-1314, May. 2009.

[158] Y. Yu, J. Dong, X. Li, and X. Zhang, "Ultra-Wideband Generation Based on Cascaded Mach-Zehnder Modulators," IEEE Photonics Technol. Lett., vol. 23, no. 23, Dec. 2011.

[159] L. Zhuang, M. R. Khan, W. Beeker, A. Leinse, R. Heideman, and C. Roeloffzen, "Novel microwave photonic fractional Hilbert transformer using a ring resonator-based optical all-pass filter," Opt. Exp., vol. 20, no. 24, pp. 26499-26510, Nov. 2012.

[160] C. Sima, J. C. Gates, C. Holmes, P. L. Mennea, M. N. Zervas, and P. G. R. Smith, "Terahertz bandwidth photonic Hilbert transformers based on synthesized planar Bragg grating fabrication,” Opt. Lett., vol. 38, no. 17, pp. 3448-3451, Sep. 2013.

[161] H. Shahoei, P. Dumais, and J. P. Yao, "Continuously tunable photonic fractional Hilbert transformer using a high-contrast germanium-doped silica-on-silicon microring resonator," Opt. Lett., vol. 39, no. 9, pp. 2778-2781, May 2014.

[162] Z. Li, Y. Han, H. Chi, X. Zhang, and J. P. Yao, "A continuously Tunable Microwave Fractional Hilbert Transformer Based on a Nonuniformly Spaced Photonic Microwave Delay-Line Filter,”J. Lightwave Technol., vol. 30, no. 12, pp. 1948-1953, Jun. 2012.

[163] Z. Li, H. Chi, X. Zhang, and J. P. Yao, "A Continuously Tunable Microwave Fractional Hilbert Transformer Based on a Photonic Microwave Delay-Line Filter Using a Polarization Modulator," IEEE Photon. Technol. Lett., vol. 23, no. 22, pp. 1694-1696, Nov. 2011.

[164] A. O.-Blanch, J. Mora, J. Capmany, B. Ortega, and D. Pastor, "Tunable radio-frequency photonic filter based on an actively modelocked fiber laser," Opt. Lett., vol. 31, no. 6, pp. 709-711, Mar. 2006.

[165] V. R. Supradeepa, C. M. Long, R. Wu. F. Ferdous, E. Hamidi, D. E. Leaird, and A. M. Weiner, "Comb-based radiofrequency photonic filters with rapid tunability and high selectivity," Nature Photonics, vol. 6, pp. 186-194, Mar. 2012.

[166] V. T.-Company, and A. M. Weiner, "Optical frequency comb technology for ultra-broadband radio-frequency photonics," Laser Photonics Rev., vol. 8, no. 3, pp. 368-393, 2014.

[167] F. Li, Y. Park, and J. Azaña, "Linear Characterization of Optical Pulses With Durations Ranging From the Picosecond to the Nanosecond Regime Using Ultrafast Photonic Differentiation,” J. Lightw. Technol., vol. 27, no. 1, pp. 4623-4633, 2009.

[168] S. Pan, J. Yao, "Optical generation of polarity- and shape-switchable ultrawideband pulses using a chirped intensity modulator and a first-order asymmetric Mach-Zehnder interferometer," Opt. Lett., vol. 34, no. 9, pp. 1312-1314, 2009.

[169] X. Li, J. Dong, Y. Yu, and X. Zhang, "A Tunable Microwave Photonic Filter Based on an All-Optical Differentiator," IEEE Photon. Technol. Lett., vol. 23, no. 22, pp. 308-310, Mar. 2011.

[170] Y. Han, Z. Li, and J. Yao, "A Microwave Bandpass Differentiator Implemented Based on a Nonuniformly-Spaced Photonic Microwave Delay-Line Filter," J. Lightw. Technol., vol. 29, no. 22, pp. 3470-3475, Nov. 2011.

[171] R. Ashrafi and J. Azaña, "Figure of merit for photonic differentiators," Opt. Exp., vol. 20, no. 3, pp. 2626-2639, Jan. 2012.

[172] F. Zeng and J. Yao, "Ultrawideband Impulse Radio Signal Generation Using a High-Speed Electrooptic Phase Modulator and a Fiber-Bragg-Grating-Based Frequency Discriminator," IEEE Photon. Technol. Lett., vol. 18, no. 19, pp. 2062-2064, Oct. 2006.

[173] P. Li, H. Chen, M. Chen, and S. Xie, "Gigabit/s Photonic Generation, Modulation, and Transmission for a Reconfigurable Impulse Radio UWB Over Fiber System," IEEE Photon. Technol. Lett., vol. 4, no. 3, pp. 805-816, Jun. 2012.

[174] Y. Yu, F. Jiang, H. Tang, L. Xu, X. Liu, J. Dong, and X. Zhang, "Reconfigurable photonic temporal differentiator based on a dualdrive Mach-Zehnder modulator," Opt. Exp., vol. 24, no. 11, pp. 11739-11748, May 2016.

[175] P. Velanas, A. Bogris, A. Argyris, and D. Syvridis, "High-Speed All-Optical First- and Second-Order Differentiators Based on Cross-Phase Modulation in Fibers,” J. Lightw. Technol., vol. 26, no. 18, pp. 3269-3276, Sep. 2008.

[176] J. Xu, X. Zhang, J. Dong, D. Liu, and D. Huang, "All-optical differentiator based on cross-gain modulation in semiconductor optical amplifier," Opt. Lett., vol. 32, no. 20, pp. 3029-3031, Oct. 2007. 
[177] J. Xu, X. Zhang, J. Dong, D. Liu, and D. Huang, "High-speed all-optical differentiator based on a semiconductor optical amplifier and an optical filter," Opt. Lett., vol. 32, no. 13, pp. 1872-1874, Jul. 2007.

[178] F. Wang, J. Dong, E. Xu, and X. Zhang, "All-optical UWB generation and modulation using SOA-XPM effect and DWDM-based multi-channel frequency discrimination," Opt. Exp., vol. 18, no. 24, pp. 24588-24594, Nov. 2010.

[179] V. Moreno, M. Rius, J. Mora, M. A. Muriel, and J. Capmany, "Integrable high order UWB pulse photonic generator based on cross phase modulation in a SOA-MZI," Opt. Exp., vol. 21, no. 19, pp. 22911-22917, Sep. 2013.

[180] Q. Wang and J. Yao, "Switchable optical UWB monocycle and doublet generation using a reconfigurable photonic microwave delay-line filter," Opt. Exp., vol. 15, no. 22, pp. 14667-14672, Oct. 2007.

[181] M. Bolea, J. Mora, B. Ortega, and J. Capmany, “Optical UWB pulse generator using an $N$ tap microwave photonic filter and phase inversion adaptable to different pulse modulation formats," Opt. Exp., vol. 17, no. 7, pp. 5023-50332, Mar. 2009.

[182] B. Mathieu, P. Melchior, A. Oustaloup, C. Ceyral, "Fractional differentiation for edge detection," Signal Processing, vol. 83, pp. 2421-2432, Nov. 2003.

[183] A. Oustaloup, F. Levron, B. Mathieu, and F. M. Nanot, "Frequency-Band Complex Noninteger Differentiator: Characterization and Synthesis," IEEE Trans. on Circuit and Systems - I: Fundamental Theory and Application, vol. 47, no. 1, pp. 25-39, Jan. 2000.

[184] M.-G. Suh, Q. Yang, K. Yang, X. Yi, and K. J. Vahala, "Microresonator soliton dual-comb spectroscopy,” Science, vol. 354, pp. 600-603, Nov. 2016.

[185] R. Slavik, Y. Park, M. Kulishov, R. Morandotti, and J. Azaña, “Ultrafast all-optical differentiator,” Opt. Exp., vol. 14 no, 22, pp. 10699, 2006.

[186] F. Liu, T. Wang, L. Qiang, T. Ye, Z. Zhang, M. Qiu, and Y. Su, "Compact optical temporal differentiator based on silicon microring," Opt. Exp., vol. 16 no. 20, pp. 15880, 2008.

[187] L. Zhang, J. Wu, X. Yin, X. Sun, P. Cao, X. Jiang, and Y. Su, “A High-Speed Second-Order Photonic Differentiator Based on Two-Stage Silicon Self-Coupled Optical Waveguide,” IEEE Photon. J., vol. 6, no. 2, pp.7900505, 2014.

[188] J. McClellan, T. W. Parks, and L. Rabiner, "A computer program for designing optimum FIR linear phase digital filters," Transactions on Audio and Electroacoustics, vol. 21, no. 6, pp. 506-526, Dec. 1973.

[189] R. C. Williamson, R. D. Esman, "RF Photonics," Journal of Lightwave Technology, vol. 26, no. 9, pp. 1145-1153, May 2008.

[190] Y. Park, T.-J. Ahn, Y. Dai, J. Yao, and J. Azaña, “All-optical temporal integration of ultrafast pulse waveforms,” Optics Express, vol. 16, no. 22, pp. 17817-17825, 2008.

[191] R. Slavík, Y. Park, N. Ayotte, S. Doucet, T.-J. Ahn, S. LaRochelle, and J. Azaña, "Photonic temporal integrator for all-optical computing," Optics Express, vol. 16, no. 22, pp. 18202-18214, 2008.

[192] M. H. Asghari, Y. Park, and J. Azaña, "New design for photonic temporal integration with combined high processing speed and long operation time window," Optics Express, vol. 19, no. 2, pp. 425-435, 2011.

[193] W. Liu, M. Li, R. S. Guzzon, E. J. Norberg, J. S. Parker, L. A. Coldren, and J. Yao, “A Photonic Temporal Integrator with an Ultra-Long Integration Time Window based on an InP-InGaAsP Integrated Ring Resonator," Journal of Lightwave Technology, vol. 32, no. 20, pp. 3654-3659, 2014.

[194] Y. Park, and J. Azaña, "Ultrafast photonic intensity integrator,” Optics Letters, vol. 34, no. 8, pp. 1156-1158, 2009.

[195] A. Malacarne, R. Ashrafi, M. Li, S. LaRochelle, J. Yao, and J. Azaña, "Single-shot photonic time-intensity integration based on a time-spectrum convolution system," Optics Letters, vol. 37, no. 8, pp. 1355-1357, 2012.

[196] J. Zhang, and J. Yao, "Microwave photonic integrator based on a multichannel fiber Bragg grating," Optics Letters, vol. 41, no. 2, pp. 273-276, 2016.

[197] M.-G. Suh, and K. J. Vahala, "Soliton microcomb range measurement," Science, vol. 359, no. 6378, pp. 884-887, 2018.

[198] A. J. Metcalf, et al., "Integrated line-by-line optical pulse shaper for high-fidelity and rapidly reconfigurable RF-filtering," Optics Express, vol. 24, no. 21, pp. 23925-23940, 2016.

[199] E. Sahin, K. J. A. Ooi, C. E. Png, and D. T. H. Tan, "Large, scalable dispersion engineering using cladding-modulated Bragg gratings on a silicon chip," Applied Physics Letters, vol. 110, pp. 161113, 2017.

[200] C. Wang, et al., "Integrated lithium niobate electro-optic modulators operating at CMOS-compatible voltages," Nature, vol. 562, pp. 101, 2018. 


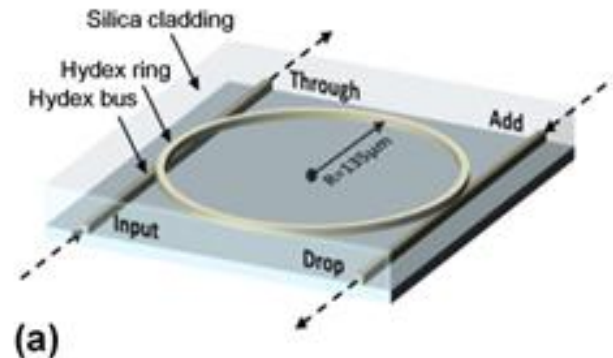

(a)

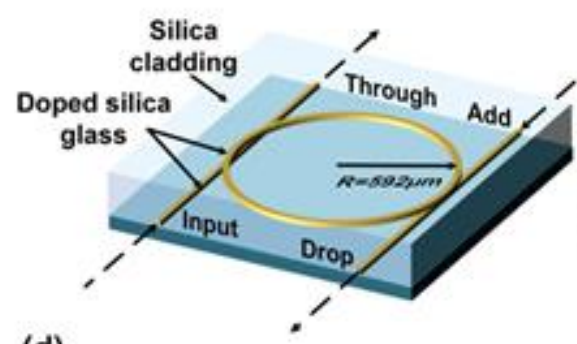

(d)

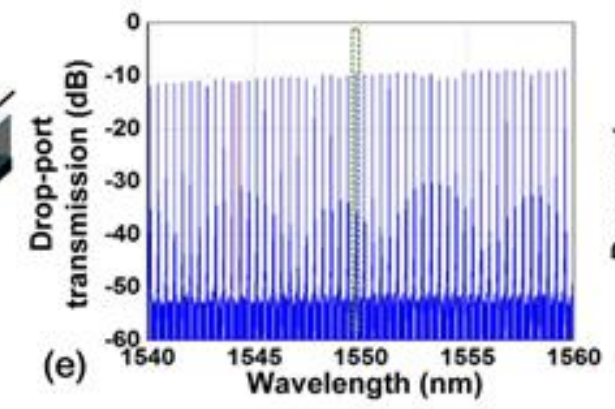

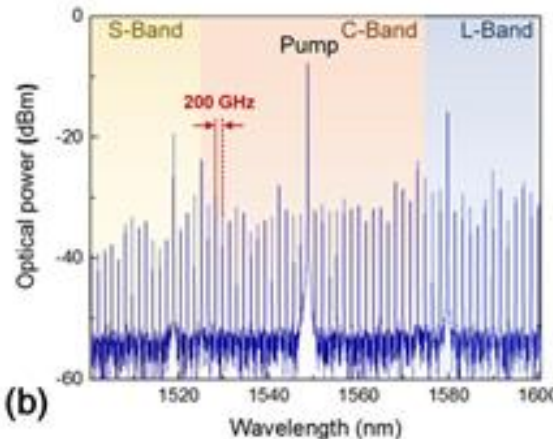
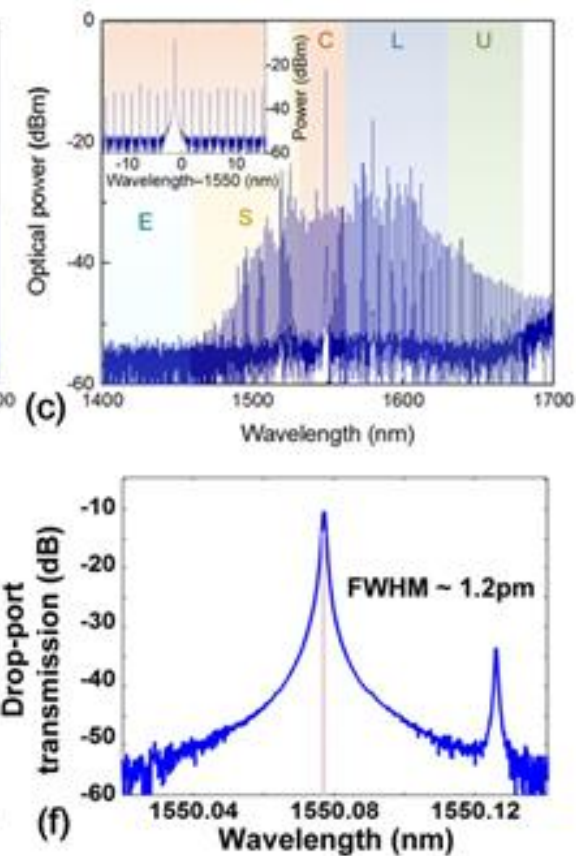

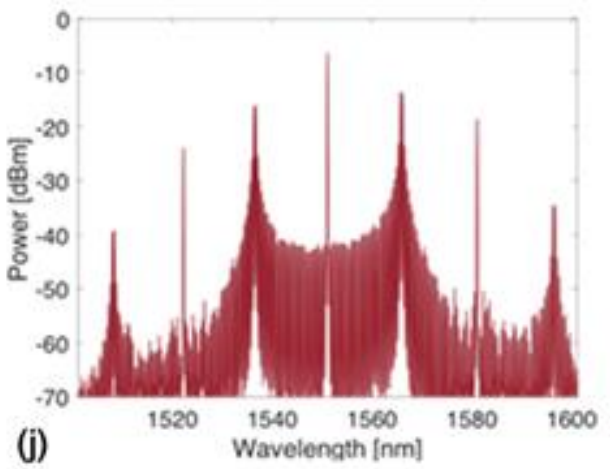

Fig. 1. Schematic illustration of the integrated MRRs for generating the Kerr micro-comb for both (a-c) 200GHz FSR combs and (d-j) 49GHz combs. (b, c) Optical spectra of the micro-combs generated by 200GHz MRR with a span of (b) $100 \mathrm{~nm}$ and (c) 300 nm. (j) Optical spectra of the micro-combs generated by $50 \mathrm{GHz}$ MRR with a span of $100 \mathrm{~nm}$. (e) Drop-port transmission spectrum of the integrated MRR with a span of $5 \mathrm{~nm}$, showing an optical free spectral range of $49 \mathrm{GHz}$. (f) A resonance at $193.294 \mathrm{THz}$ with full width at half maximum (FWHM) of $124.94 \mathrm{MHz}$, corresponding to a quality factor of $1.549 \times 10^{6}$.

\section{Figure 1}



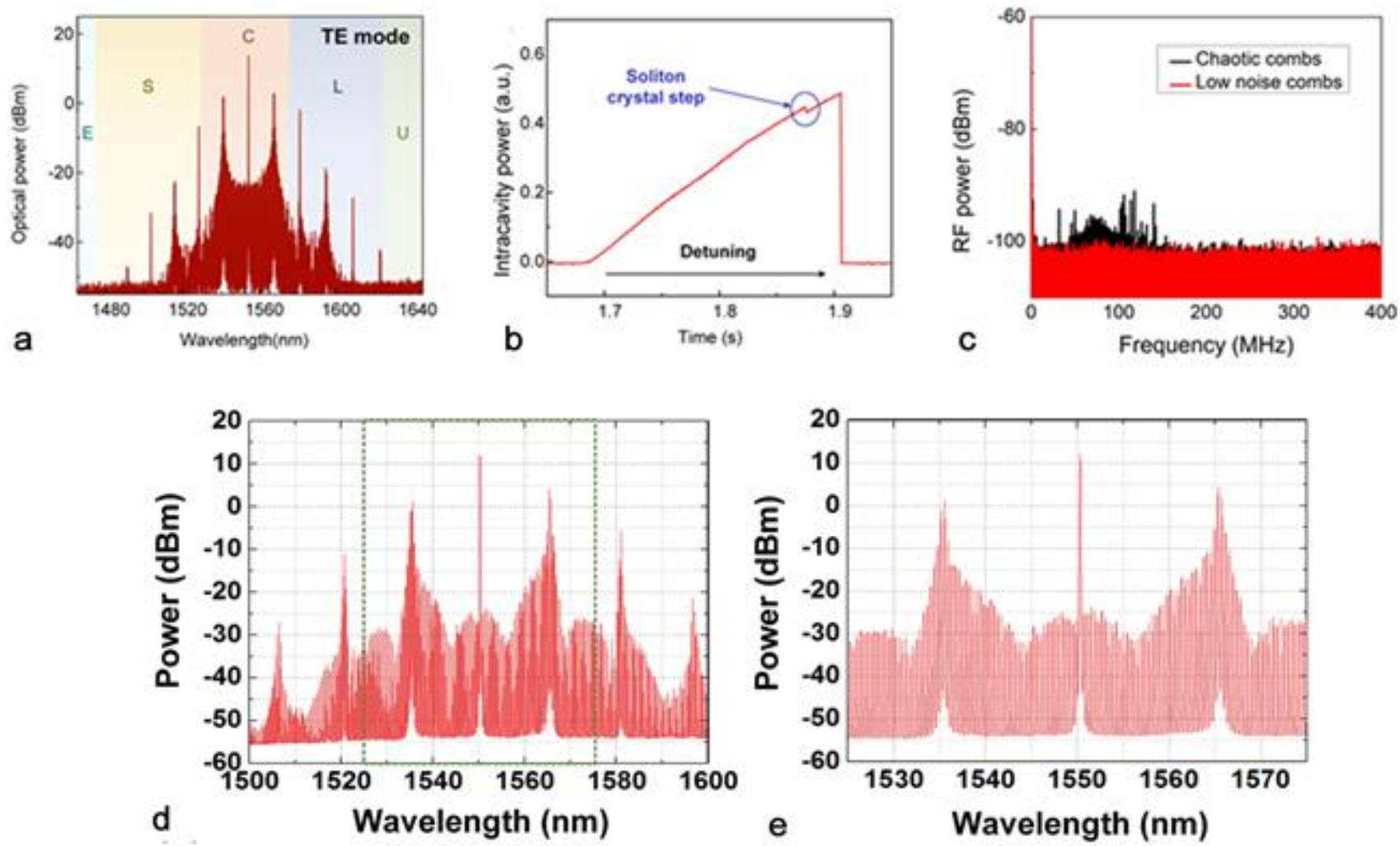

Fig. 2. (a, d, e) Optical spectra of various soliton crystal micro-combs. (b) Optical power output versus pump tuning, showing the very small power jump at the onset of soliton crystal combs. (c) Transition from high RF noise chaotic state to low noise state of the soliton crystal comb. 

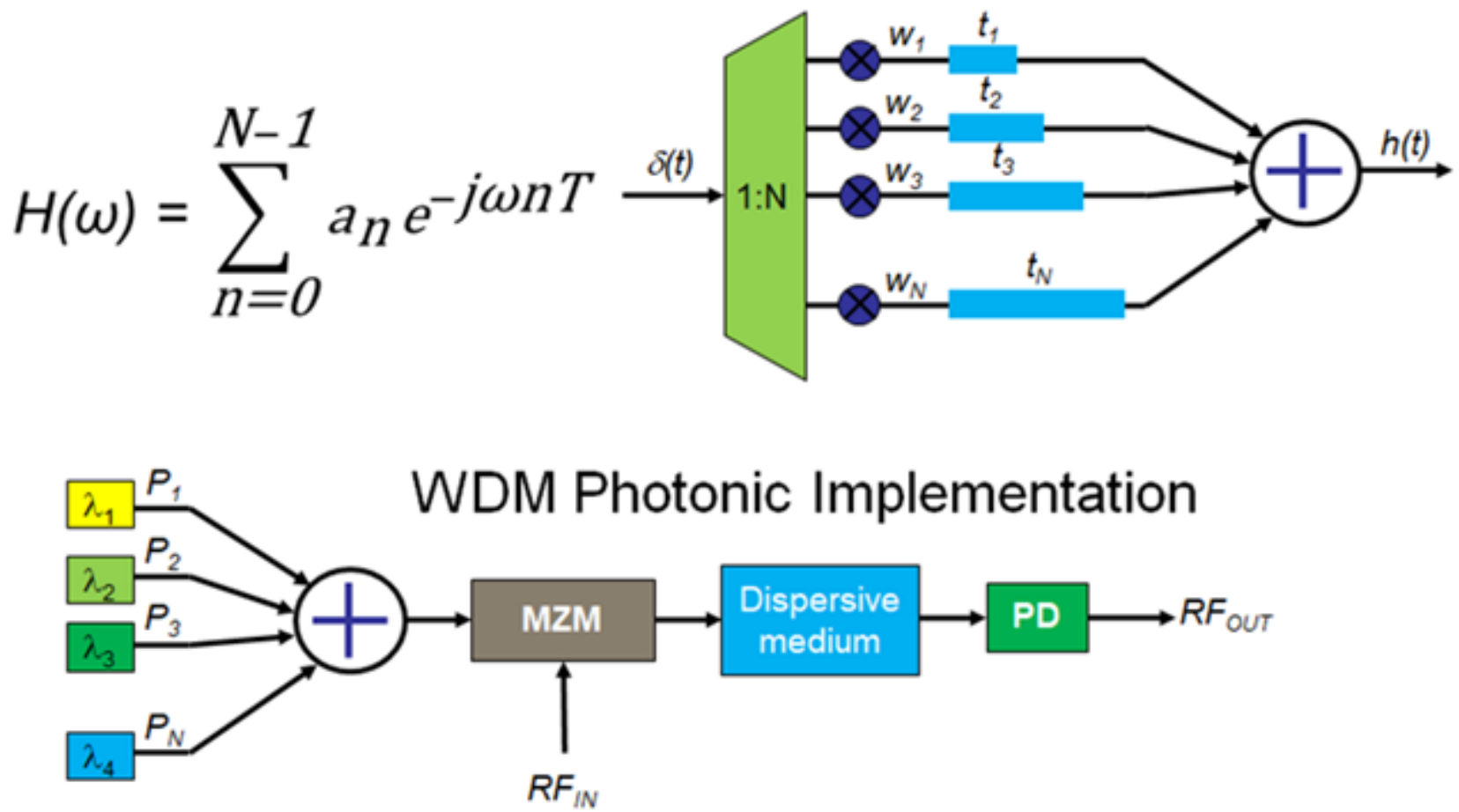

Fig. 3. Theoretical schematic of the principle of transversal filters using wavelength multiplexing. MZM: Mach-Zehnder modulators. PD: photo-detector.

Figure 3 

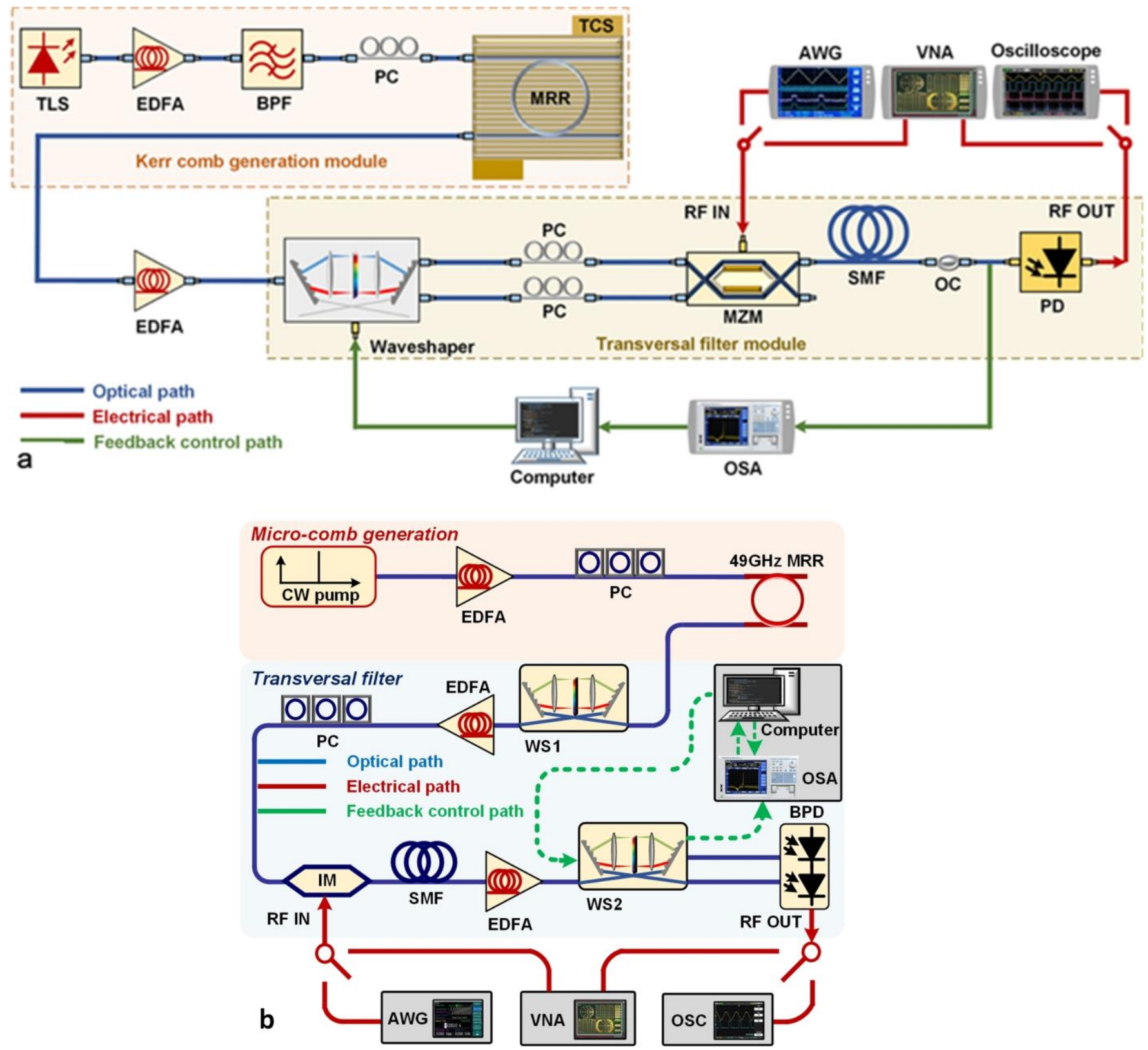

Fig. 4. Experimental schematic for RF transversal filters based on $200 \mathrm{GHz}$ microcomb (top) and $49 \mathrm{GHz}$ microcomb (bottom). TLS: tunable laser source. EDFA: erbium-doped fiber amplifier. PC: polarization controller. BPF: optical bandpass filter. TCS: temperature control stage. MRR: micro-ring resonator. WS: WaveShaper. OC: optical coupler. SMF: single mode fibre. OSA: optical spectrum analyzer. AWG: arbitrary waveform generator. VNA: vector network analyser. PD: photodetector. BPD: balanced photodetector. 

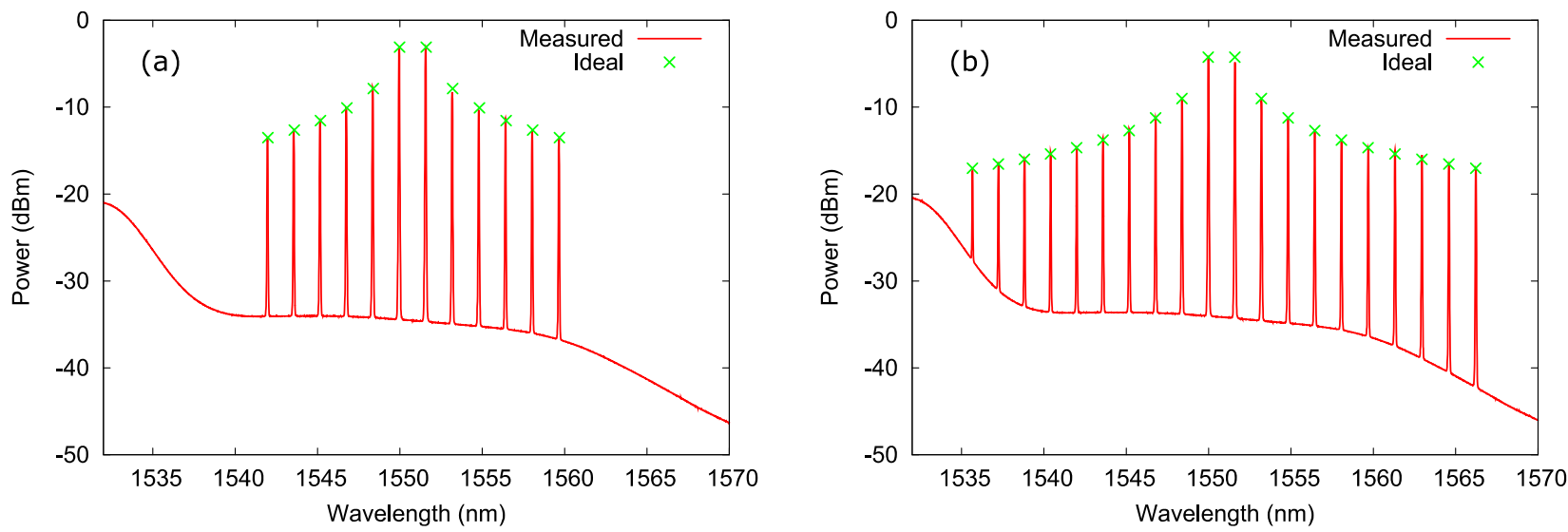

Fig. 5. Shaped optical spectra showing the weight of each tap for: (a) 12 tap filter, and (b) 20 tap filter

Figure 5 

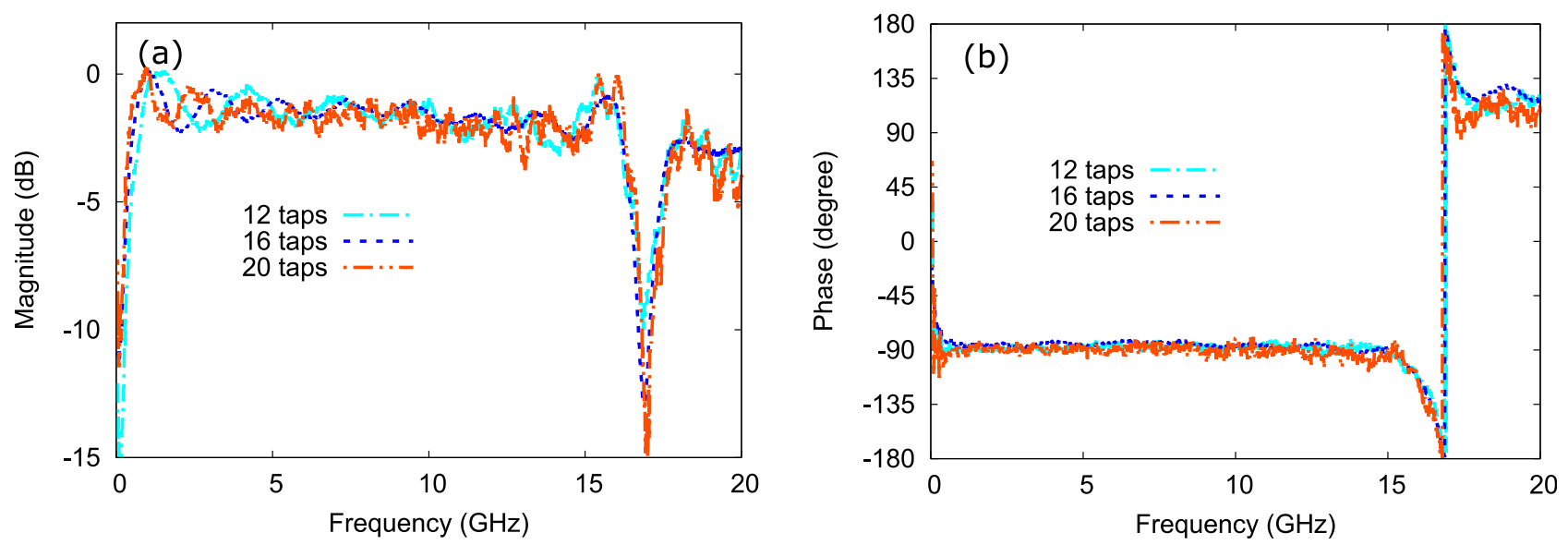

Fig. 6. Measured system RF frequency response for different number of filter taps: (a) amplitude; and (b) phase response.

\section{Figure 6}




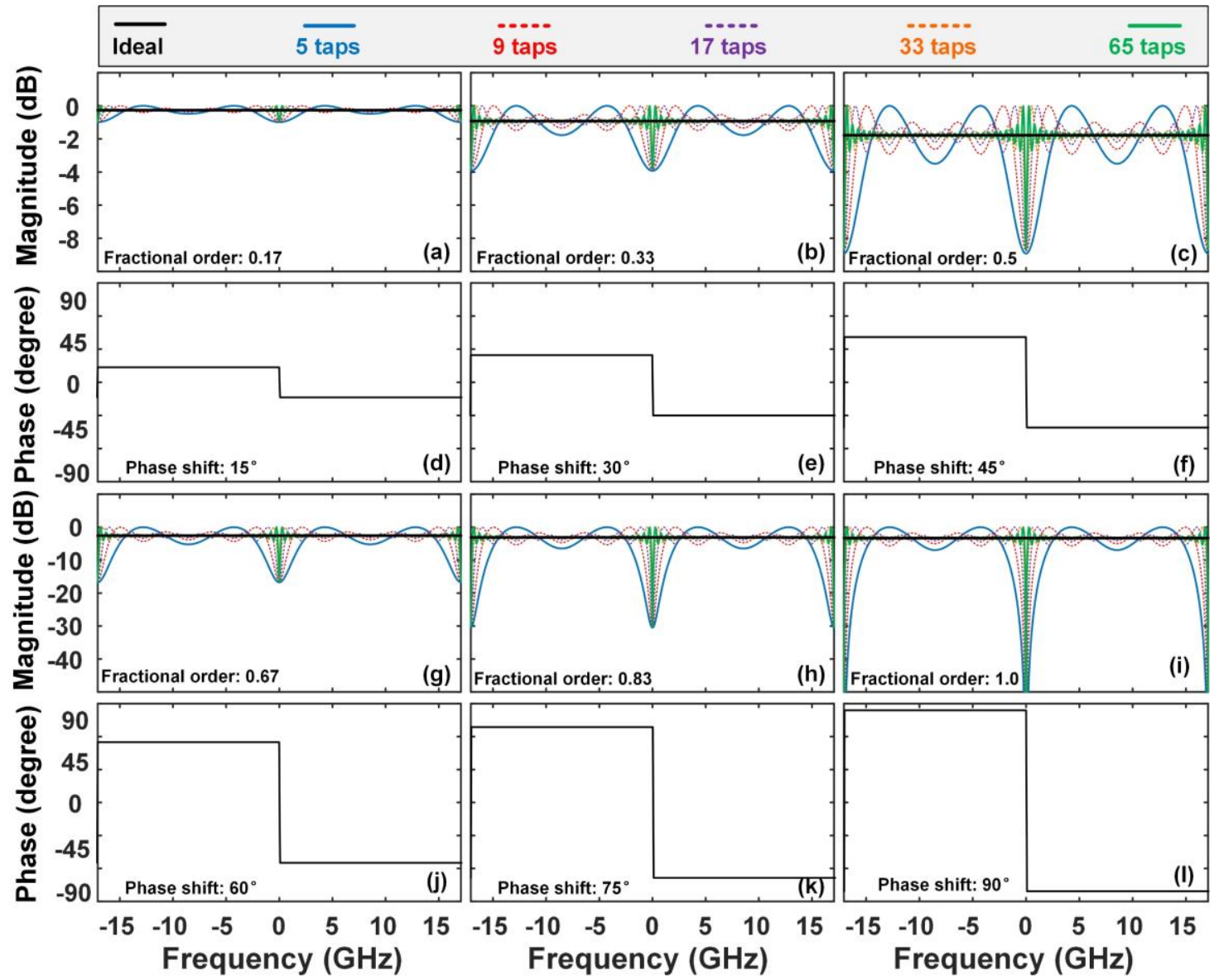

Fig. 7. Theoretical RF amplitude and phase response of FHTs with (a, d) $15^{\circ}$, (b, e) $30^{\circ}$, (c, f) $45^{\circ},(\mathrm{g}, \mathrm{j}) 60^{\circ}$, (h, k) $75^{\circ}$, and (i, l) $90^{\circ}$ phase shifts. The amplitude of the fractional Hilbert transformers designed based on Eq. (3) (colour curves) are shown according to the number of taps employed. 


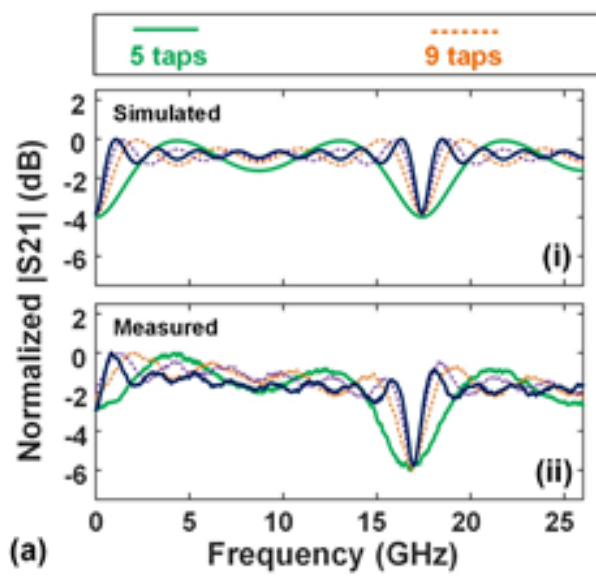

(i).
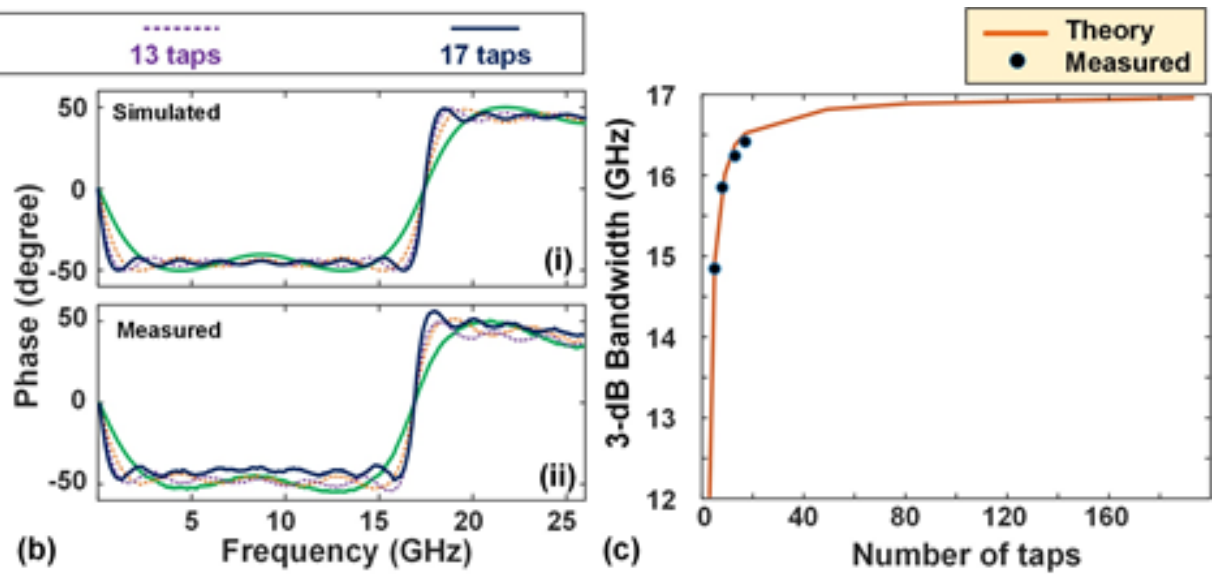

Fig. 8. (a) and (b) Simulated and measured amplitude and phase response for different number of taps for a phase shift of $45^{\circ}$. (c) Simulated and experimental results of 3-dB bandwidth with different taps for a phase shift of $45^{\circ}$.

\section{Figure 8}



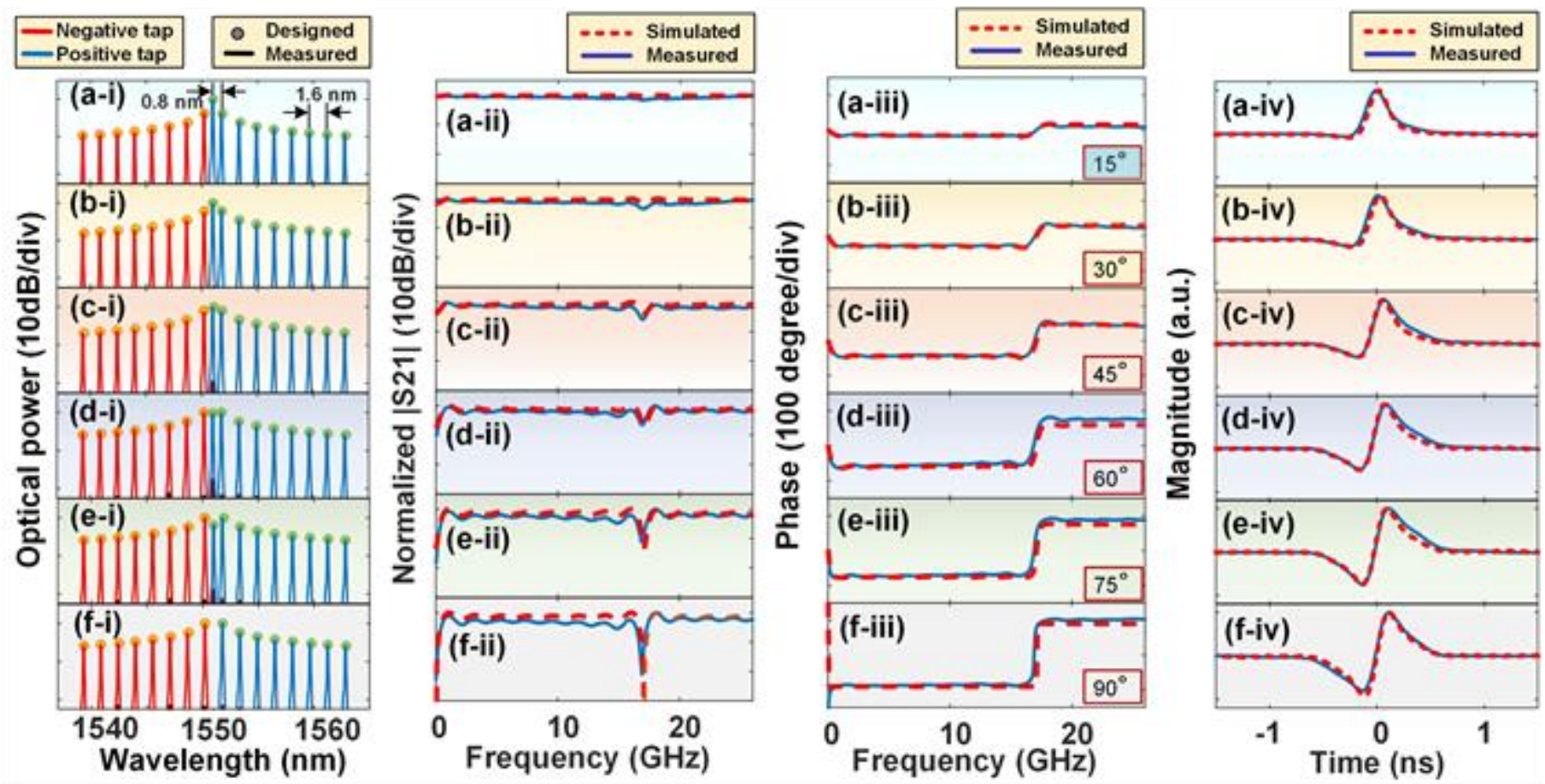

Fig. 9. Simulated (dashed curves) and experimental (solid curves) results of FHT with various phase shifts of (a) $15^{\circ}$, (b) $30^{\circ}$, (c) $45^{\circ}$, (d) $60^{\circ}$, (e) $75^{\circ}$, and (f) $90^{\circ}$. (i) Optical spectra of the shaped micro-comb corresponding with positive and negative tap weights (ii) RF amplitude responses with fractional orders of $0.166,0.333,0.5,0.667,0.833$, and 1. (iii) RF phase responses with phase shifts of $15^{\circ}, 30^{\circ}, 45^{\circ}, 60^{\circ}, 75^{\circ}$ and $90^{\circ}$. (iv) Output temporal intensity waveforms after the FHT. 

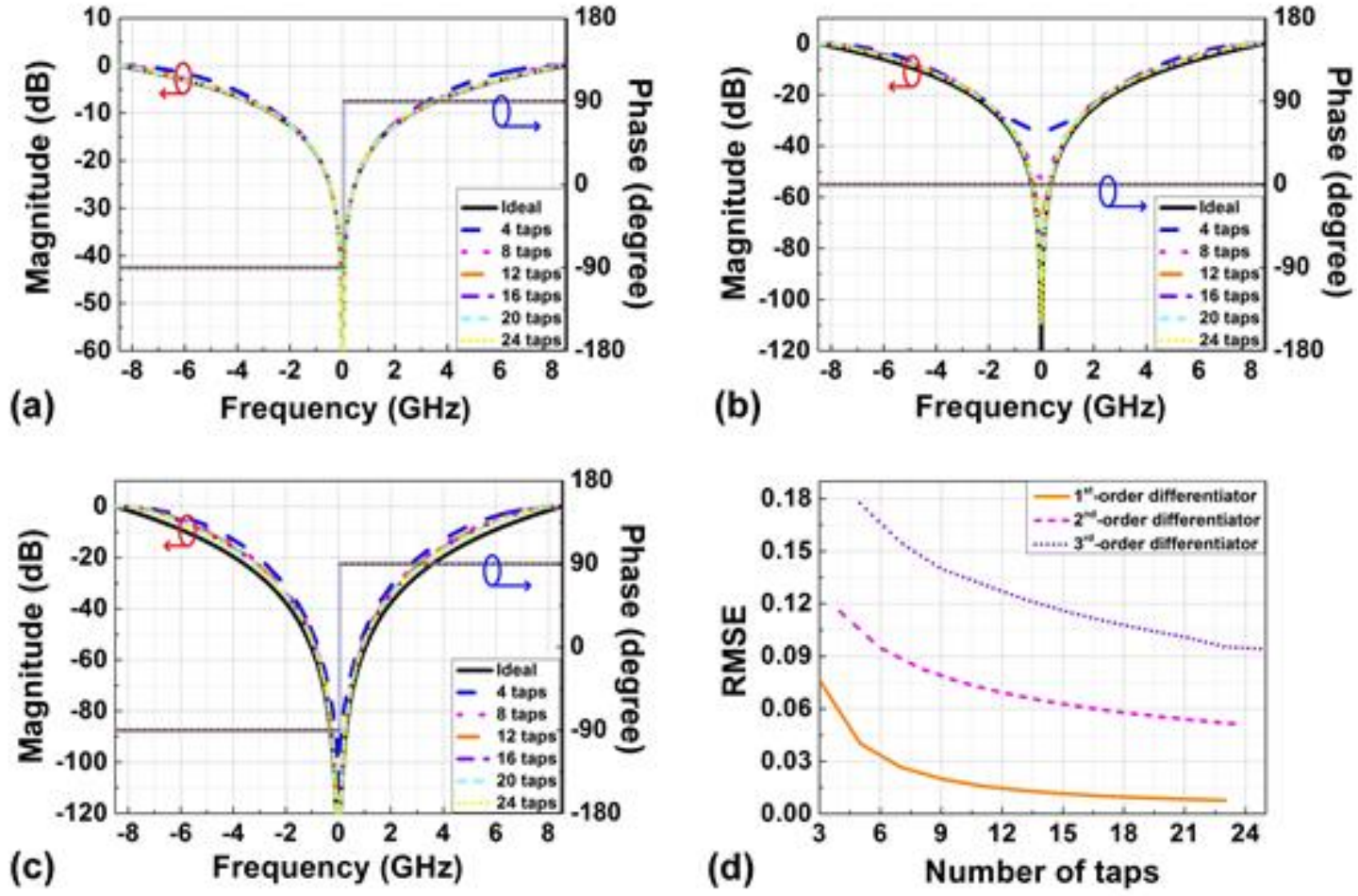

Fig. 10. Simulated RF amplitude and phase responses of the (a) first-, (b) second-, and (c) third-order temporal differentiators. (d) RMSEs between calculated and ideal RF amplitude responses of the first-, second-, and third-order intensity differentiators as a function of the number of taps. 

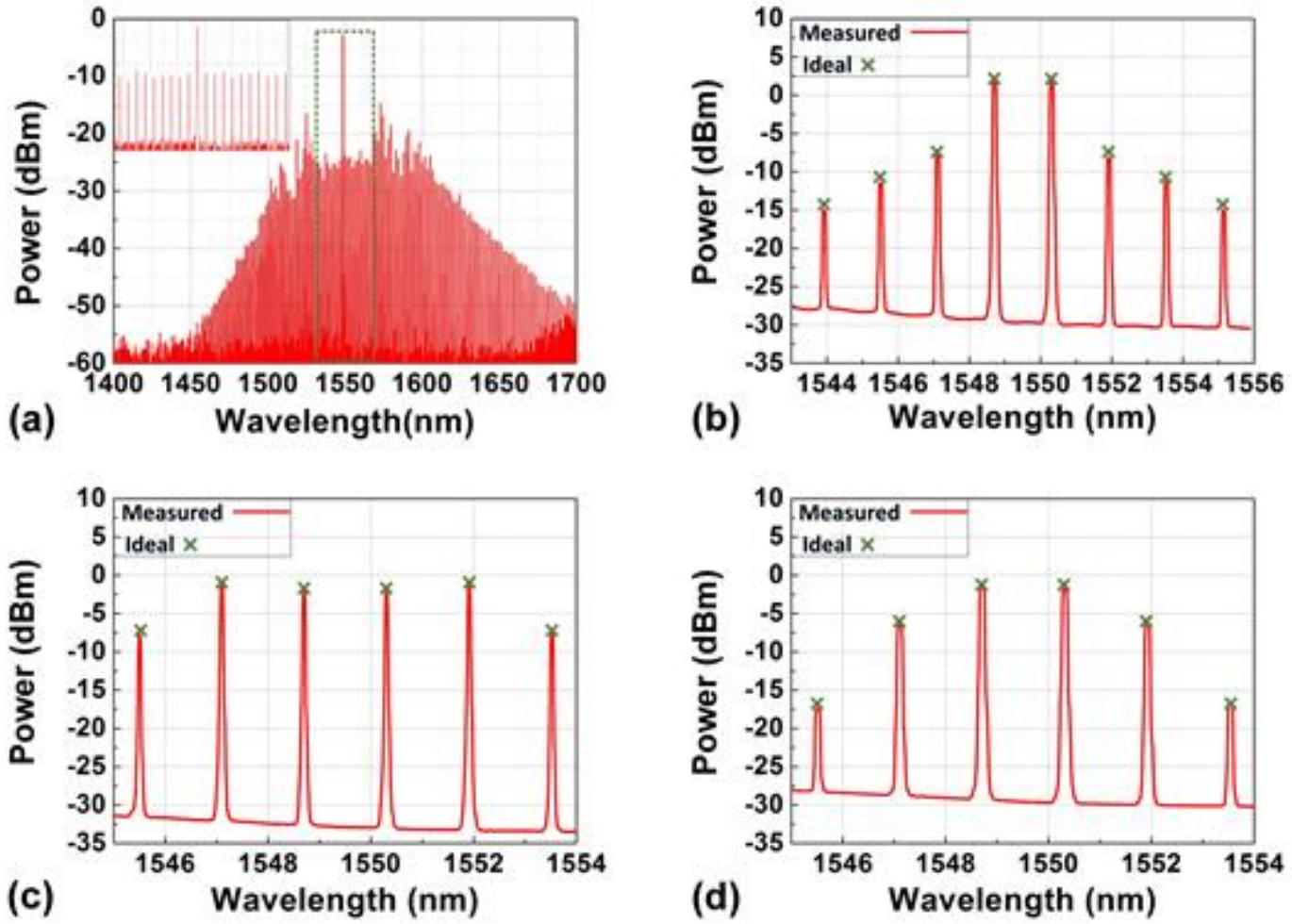

Fig. 11. (a) Optical spectrum of the generated Kerr comb in a 300-nm wavelength range. Inset shows a zoom-in spectrum with a span of $\sim 32 \mathrm{~nm}$. (b)-(d) Measured optical spectra (red solid) of the shaped optical combs and ideal tap weights (green crossing) for the first-, second-, and third-order intensity differentiators. 

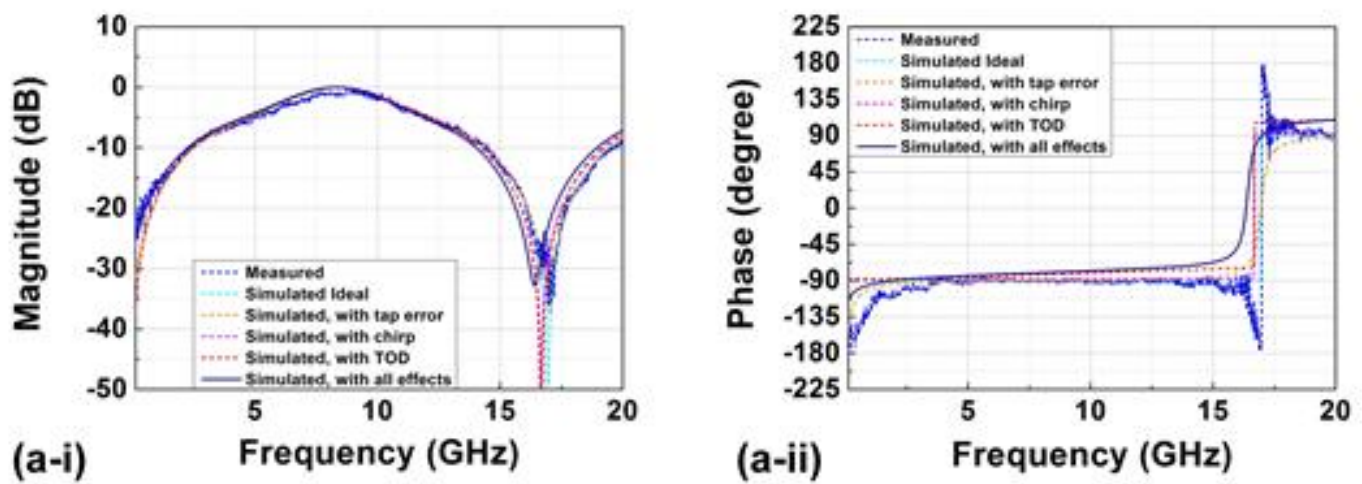

(a-i)

Frequency $(\mathrm{GHz})$
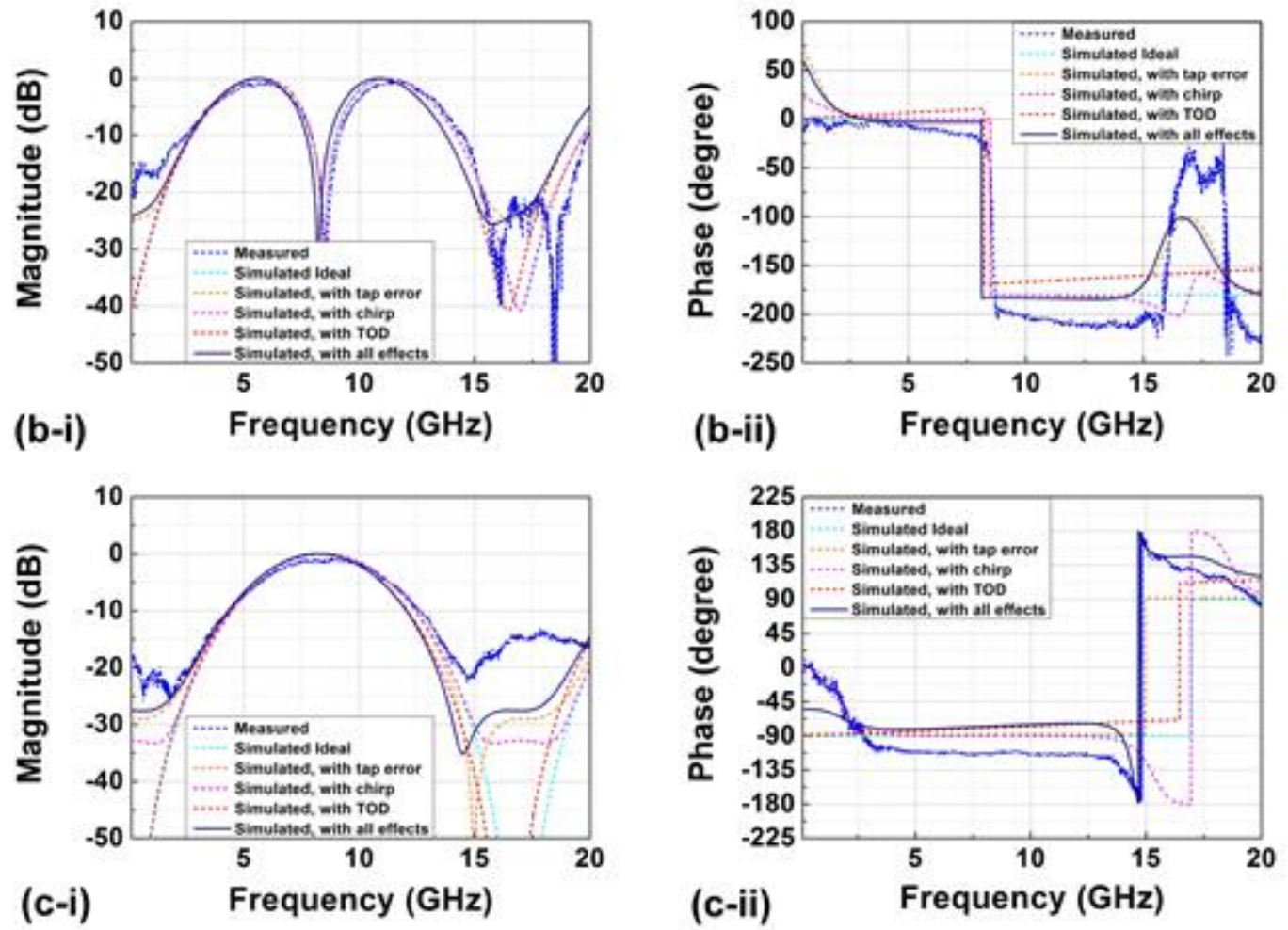

Fig. 12. Measured and simulated RF amplitude and phase responses of (a-i)-(a-ii) the first-order, (b-i)(b-ii) second-order, and (c-i)-(c-ii) third-order intensity differentiators. 

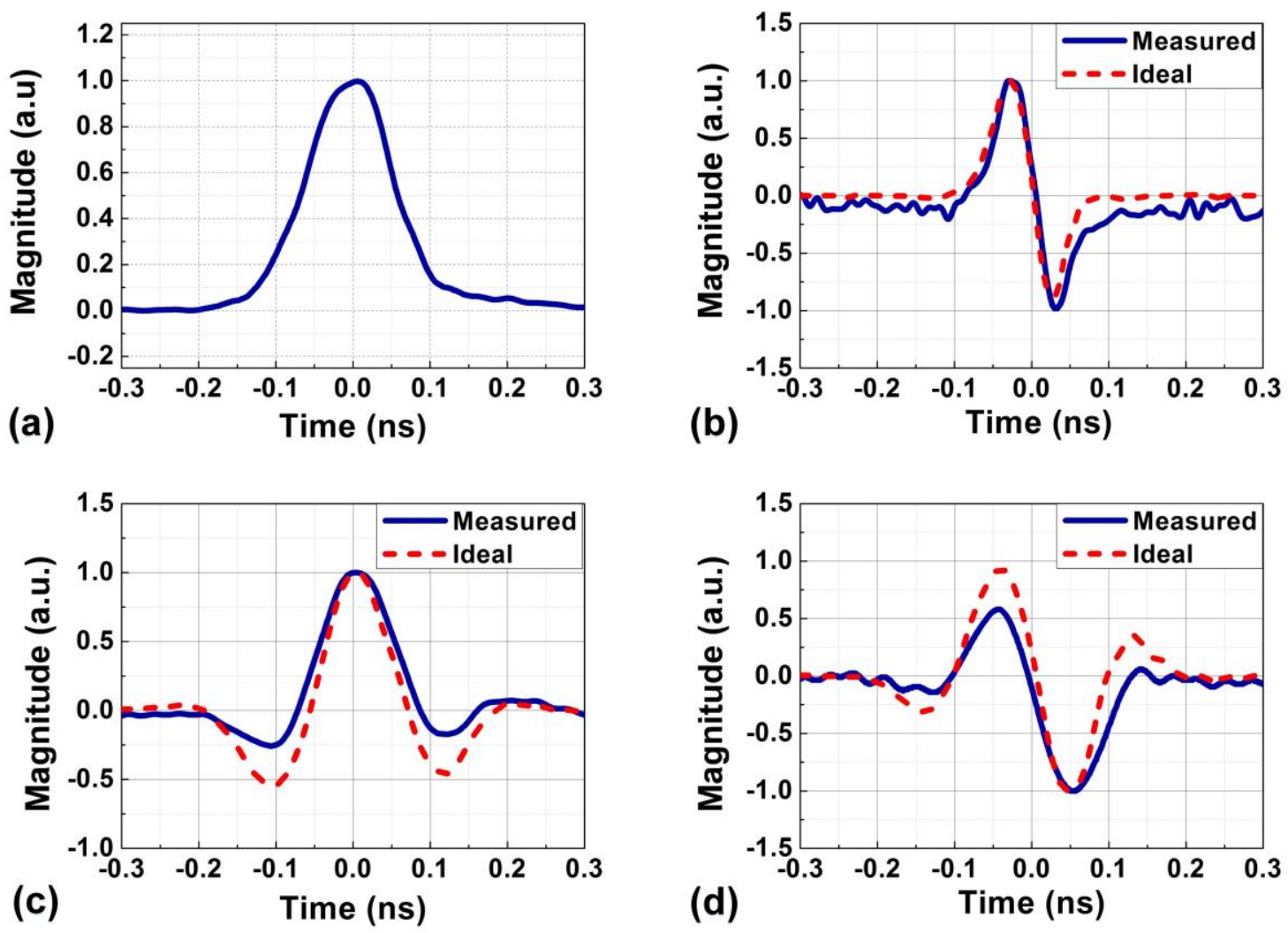

Fig.13. (a) Measured temporal waveforms of a Gaussian input pulse. Theoretical (red dashed) and experimental (blue solid) responses of the (b) first-, (b) second-, and (c) third-order differentiators.

Figure 13 


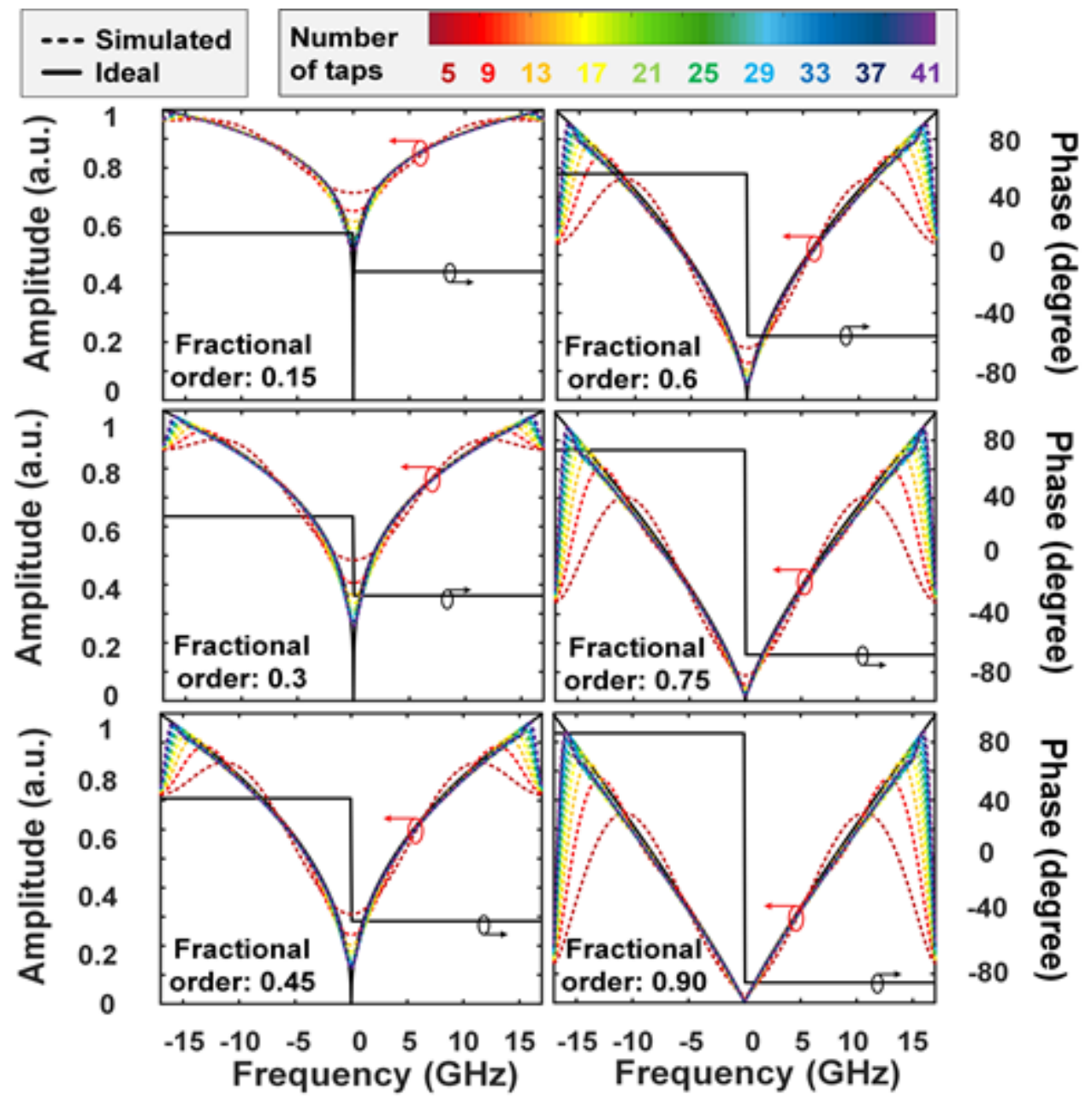

Fig. 14. Simulated transfer function of different fractional differentiation orders with varying number of taps.

Figure 14 

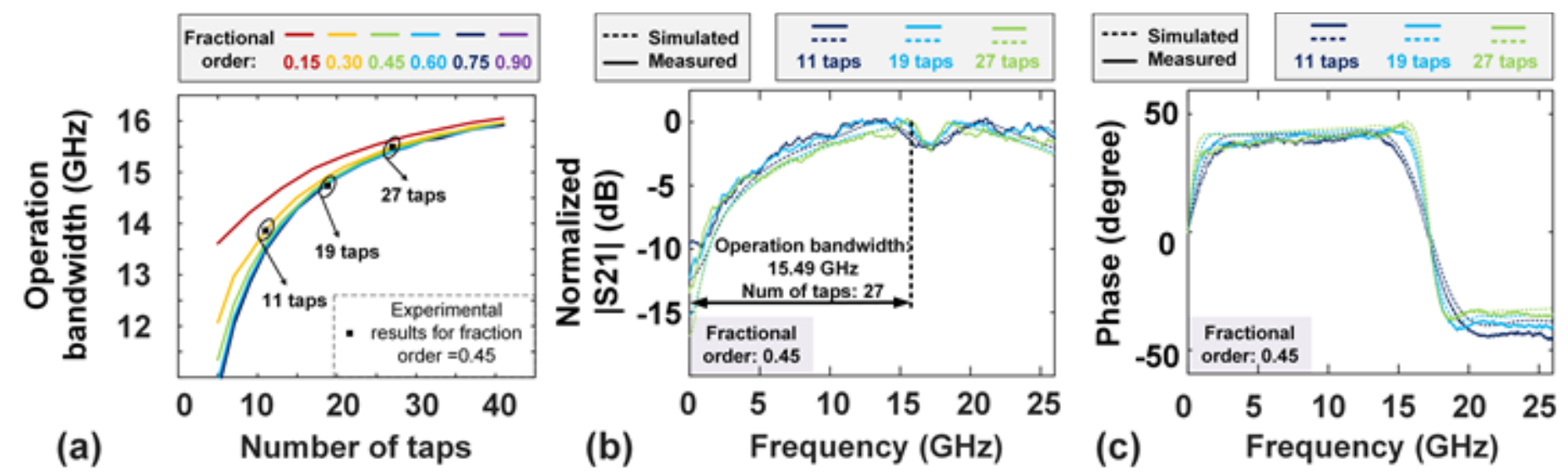

Fig. 15. (a) Extracted relationship between the number of taps and operation bandwidth. (b, c) Experimentally demonstrated fractional differentiator with varying number of taps.

\section{Figure 15}




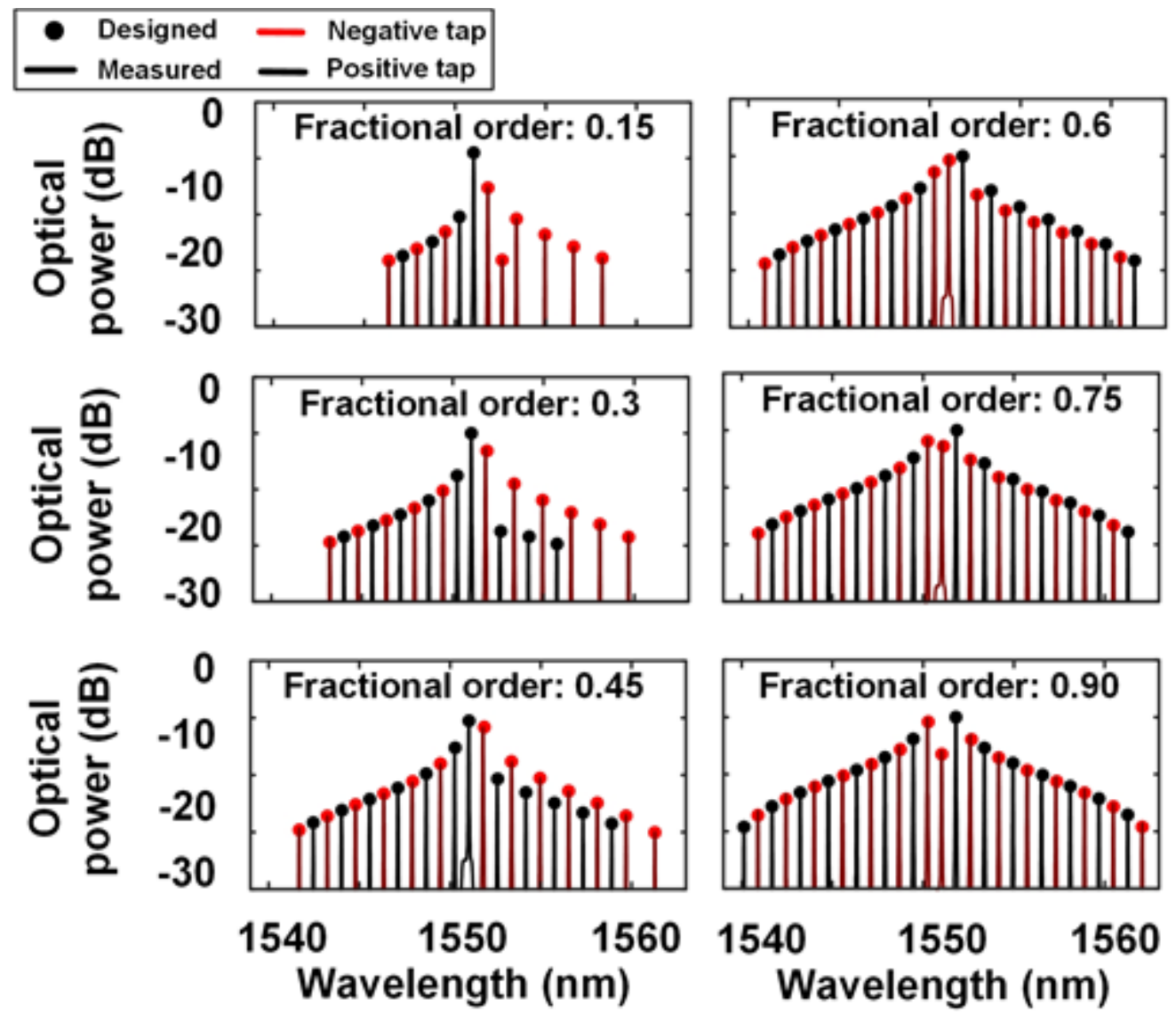

Fig. 16. Optical spectra of the shaped micro-comb for different fractional orders.

Figure 16 


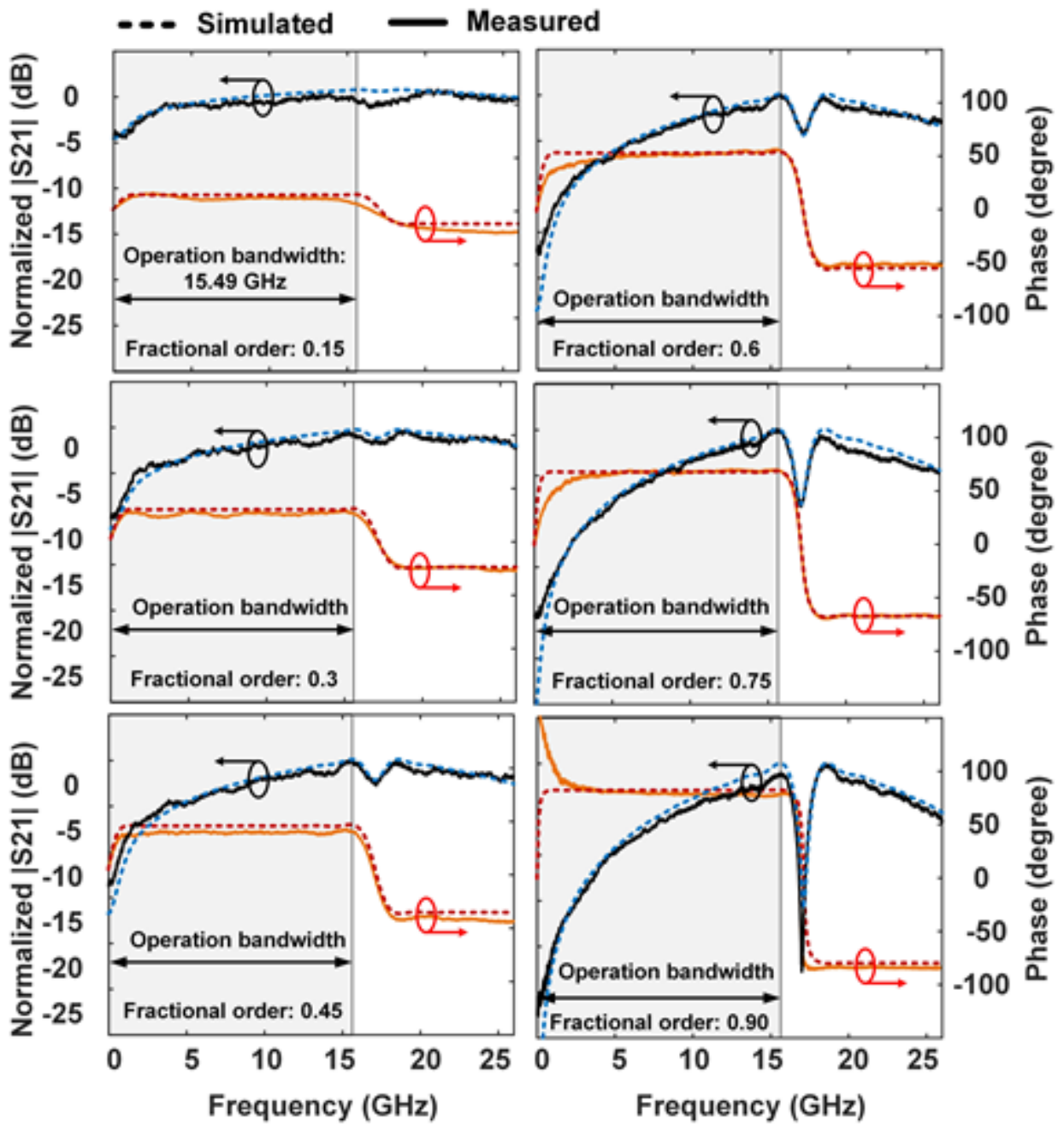

Fig. 17. Simulated and measured the transmission response of the fractional differentiator at different orders ranging from 0.15 to 0.90 . 

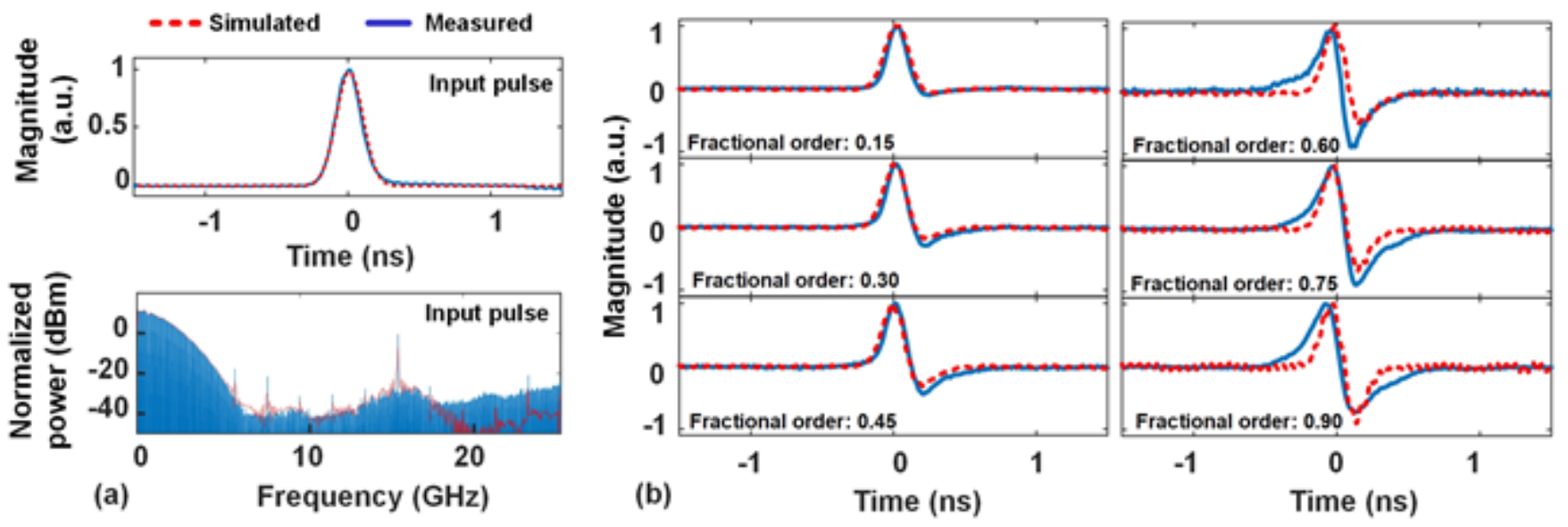

Fig. 18. Simulated and measured RF Gaussian pulse output temporal intensity waveform after the fractional differentiator.

Figure 18 


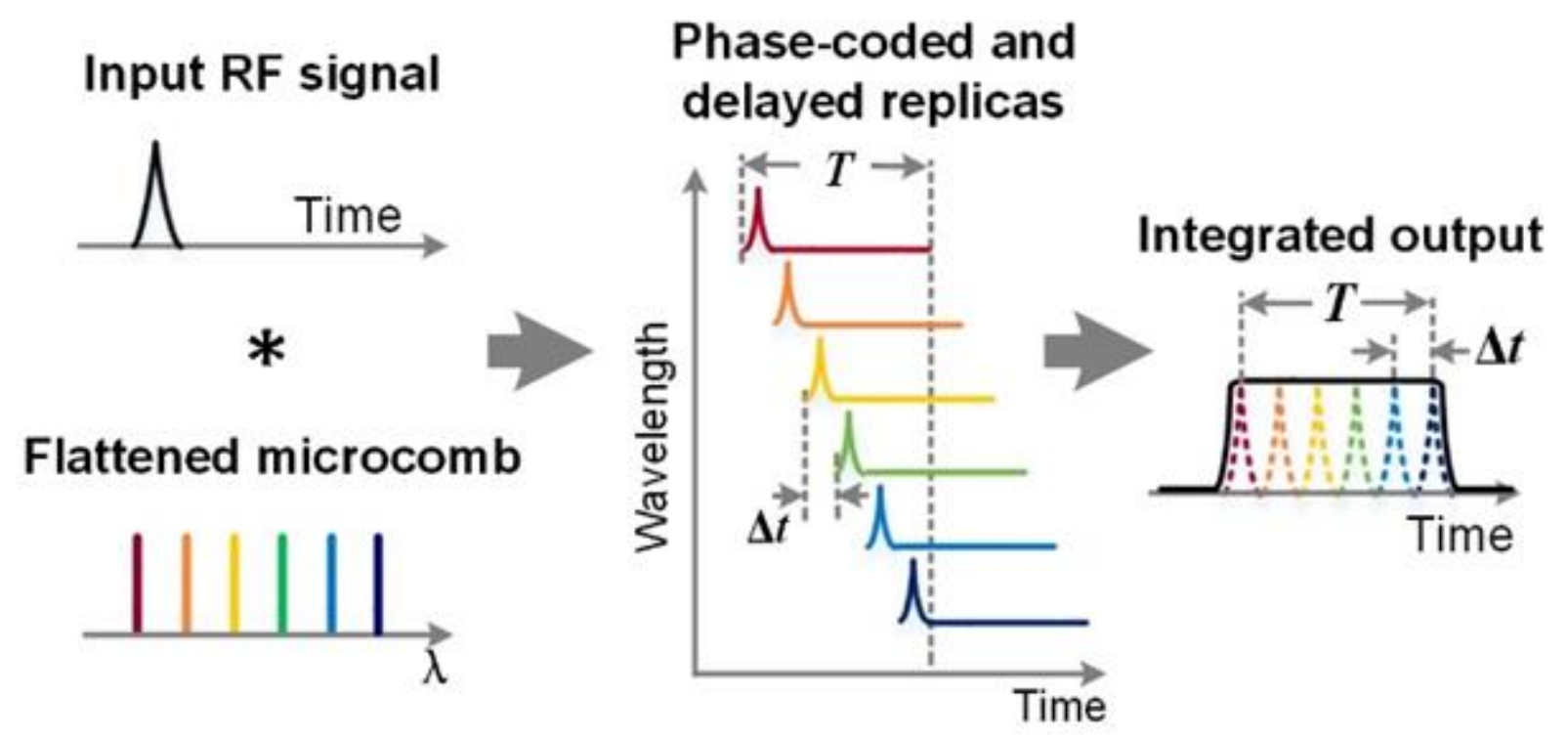

Fig. 19. Schematic diagram of the photonic RF integration.

Figure 19 

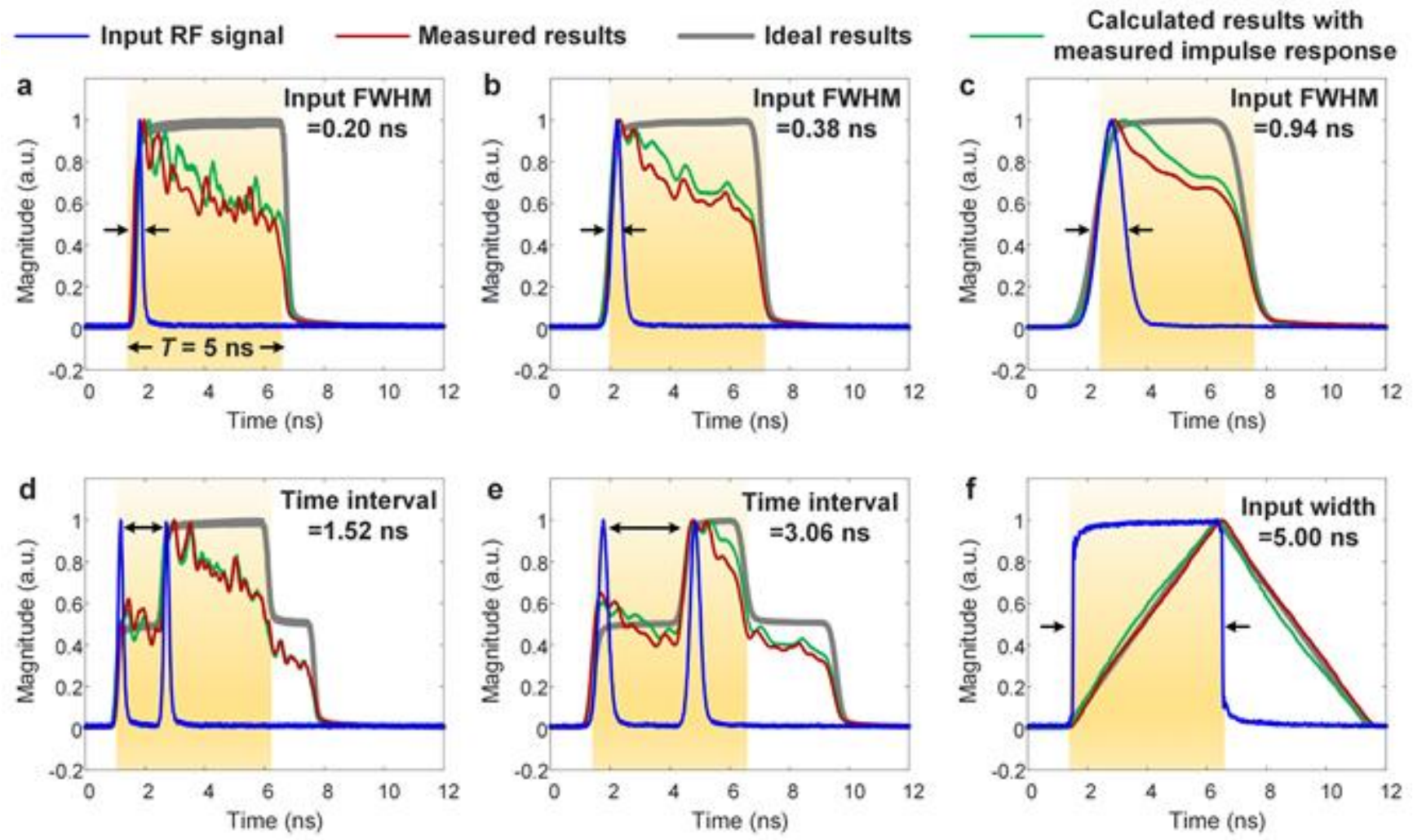

Fig. 20. Experimental results of the micro-comb-based RF integrator after comb optical power shaping for input (a-c) Gaussian pulses with FWHM of $0.20,0.38$ and $0.94 \mathrm{~ns}$, (d-e) dual Gaussian pulses with time intervals of 1.52 and $30.6 \mathrm{~ns}$, and (f) a triangular waveform with a width of $5.00 \mathrm{~ns}$. The blue curves denote the input signal, the red curves denote the measured integration results, the gray curves denote the ideal integration results, and the green curves denote the integration results calculated with the measured impulse response of the system. 

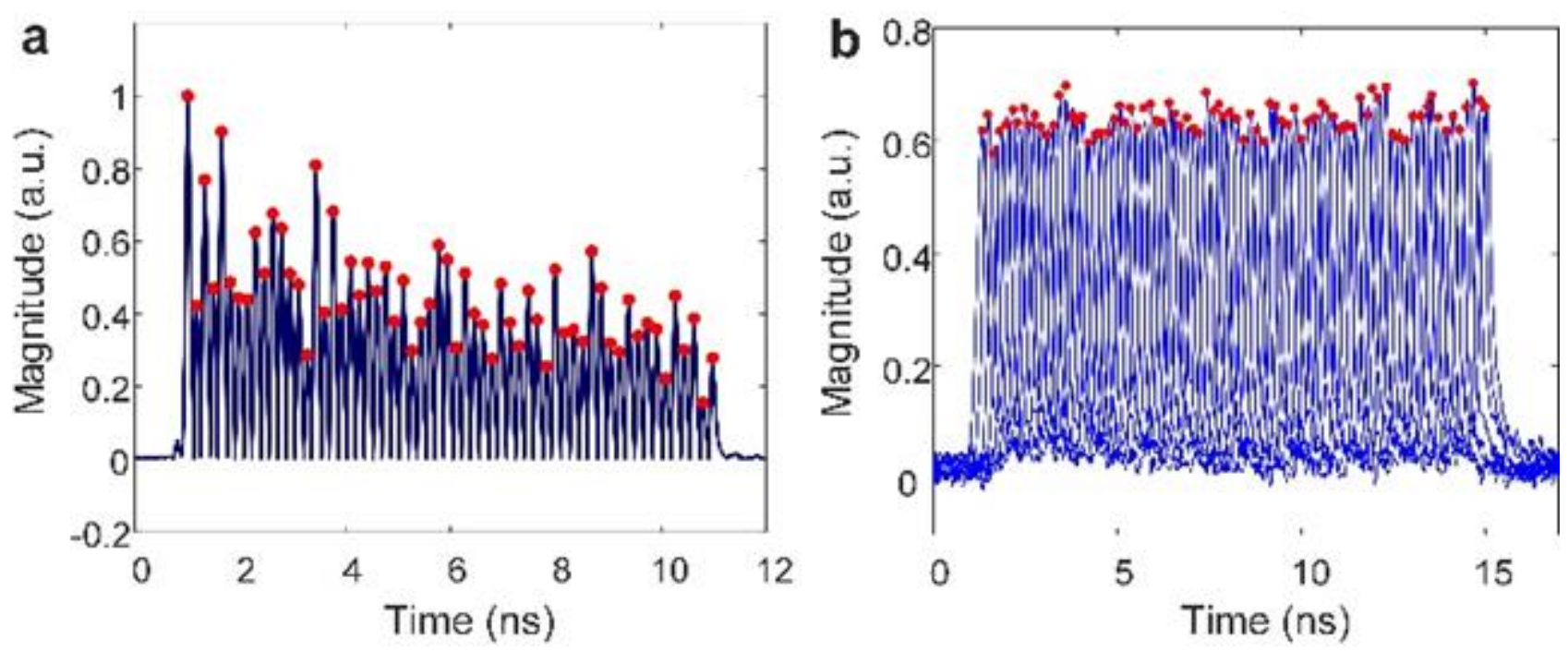

Fig. 21. Measured impulse response of the integrator (a) after comb optical power shaping and (b) after impulse response shaping using a Gaussian RF input pulse.

Figure 21 


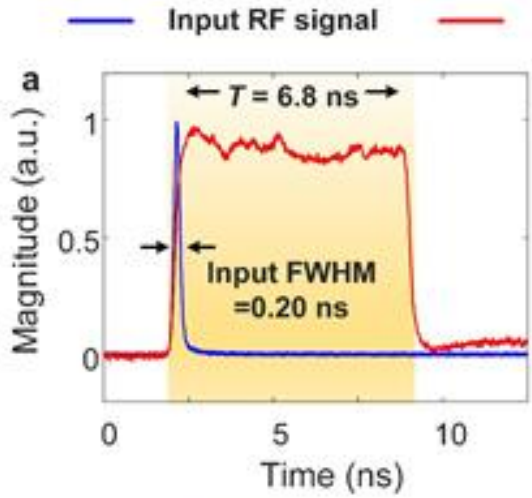

Measured integration results
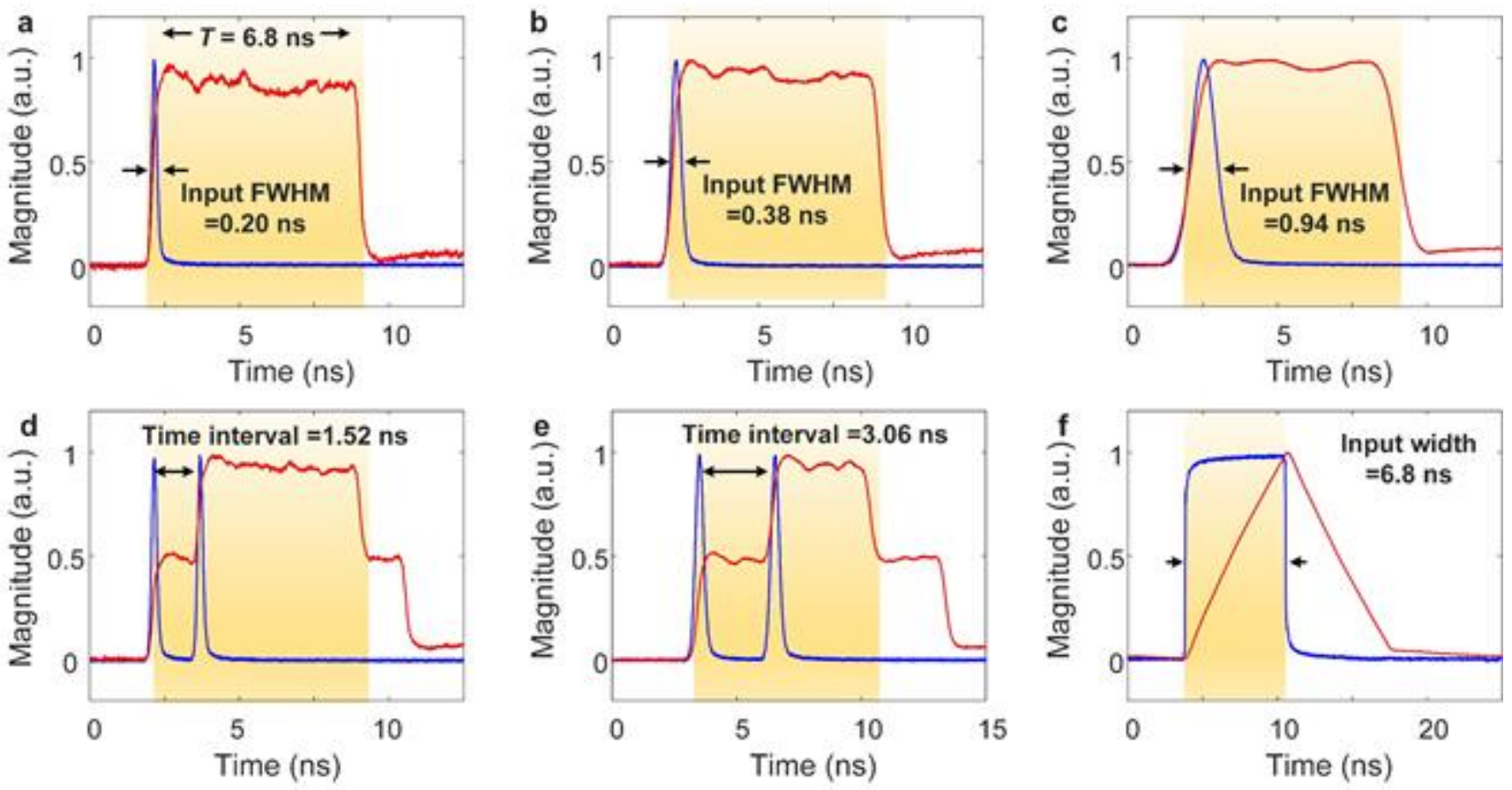

Fig. 22. Experimental results of the micro-comb-based RF integrator after impulse response shaping for input (a-c) Gaussian pulses with FWHM of 0.20, 0.38 and $0.94 \mathrm{~ns}$, (d-e) dual Gaussian pulses with time intervals of 1.52 and $3.06 \mathrm{~ns}$, and (f) a triangular waveform with a width of $5.00 \mathrm{~ns}$. The blue curves denote the input signal, the red curves denote the measured integration results. 